\title{
Diffractive dijet production: Breakdown of factorization
}

\author{
B. Z. Kopeliovich, ${ }^{1, *}$ Roman Pasechnik, ${ }^{2,3, \dagger}$ and I. K. Potashnikova ${ }^{1, \$}$ \\ ${ }^{1}$ Departamento de Física, Universidad Técnica Federico Santa María, \\ Centro Científico-Tecnológico de Valparaíso, Casilla 110-V, Valparaíso, Chile \\ ${ }^{2}$ Department of Astronomy and Theoretical Physics, Lund University, SE-223 62 Lund, Sweden \\ ${ }^{3}$ Nuclear Physics Institute ASCR, 25068 Řež, Czech Republic
}

(Received 15 July 2018; published 19 December 2018)

\begin{abstract}
We analyze the origin of dramatic breakdown of diffractive factorization, observed in single-diffractive (SD) dijet production in hadronic collisions. One of the sources is the application of the results of measurements of the diagonal diffractive deep inelastic scattering to the off-diagonal hadronic diffractive process. The suppression caused by a possibility of inelastic interaction with the spectator partons is calculated at the amplitude level, differently from the usual probabilistic description. It turns out, however, that interaction with the spectator partons not only suppresses the SD cross section, but also gives rise to the main mechanism of SD dijet production, which is another important source of factorization failure. Our parameter-free calculations of SD-to-inclusive cross section ratio, performed in the dipole representation, agrees with the corresponding CDF Tevatron (Run II) data at $\sqrt{s}=1.96 \mathrm{TeV}$ in the relevant kinematic regions. The energy and hard scale dependences demonstrate a trend, opposite to the factorization-based expectations, similarly to the effect observed earlier in diffractive Abelian radiation.
\end{abstract}

DOI: 10.1103/PhysRevD.98.114021

\section{INTRODUCTION}

\section{A. Why diffractive factorization fails}

Hadronic diffraction at high energies provides opportunities for a better understanding of an interplay between short- and long-range QCD interactions. Diffractive processes, even diffractive deep inelastic scattering (DIS) at high $Q^{2}[1]$, are typically dominated by soft interactions that are difficult to predict from first-principle QCD [2,3]. A special class of hard diffractive reactions that necessarily involves a large rapidity gap and hard interactions, in particular high- $p_{T}$ particle production, have been intensively studied over past two decades.

Factorization of short and long distance interactions has been expected to hold for this class of processes in analogy to inclusive reactions. It looked natural to assume that one can measure the PDFs of the Pomeron in the diffractive DIS, and assuming their universality, apply the results to hard diffractive processes in hadronic collisions [4]. However, CDF data [5] on diffractive dijet production

\footnotetext{
*bzk@mpi-hd.mpg.de

'Roman.Pasechnik@thep.lu.se

*irina.potashnikova@usm.cl
}

Published by the American Physical Society under the terms of the Creative Commons Attribution 4.0 International license. Further distribution of this work must maintain attribution to the author(s) and the published article's title, journal citation, and DOI. Funded by SCOAP ${ }^{3}$. revealed a dramatic, order of magnitude, breakdown of such a diffractive factorization. The mechanism, leading to failure of factorization, is usually related to the presence of spectator partons in hadronic collisions. Sometimes it results in an additional suppression factor, called rapidity gap survival probability. The diffraction amplitude, however, is a linear combination of elastic amplitudes of different Fock components of the proton, which contain rapidity gaps by default.

Other mechanisms of factorization breaking, related to the multigluon Pomeron structure were proposed in [6-8]. Differently from diffractive DIS, in hadronic collisions the Pomeron can be attached simultaneously to the projectile gluon and to the produced parton pair. In other words, the back-to-back high- $p_{T}$ pair, which has a lifetime substantially shorter than the projectile gluon in the incoming hadron, may be produced during the interaction.

A novel mechanism of diffractive factorization breaking was proposed in Refs. $[9,10]$ for the Drell-Yan process, for gauge and Higgs bosons in Refs. [11,12], and for diffractive heavy flavor production in Ref. [13]. For a review on breakdown of diffractive factorization in hadronic collisions, see Refs. [14,15].

The main reason of nonuniversality of the diffractive structure functions, measured in DIS, is the principal difference between the diagonal and off-diagonal diffractive processes. Diffractive DIS, $\gamma^{*}+p \rightarrow X+p$, is predominantly diagonal (elastic $\bar{q} q+p \rightarrow \bar{q} q+p$ ), so one should not apply the results of such measurements to the 
off-diagonal diffractive processes (e.g., dijets) in hadronic collisions. In terms of the Regge approach diffraction is related to the Pomeron exchange, but the Pomerons in the above two cases are different. Even if the Pomeron were a true Regge pole with a universal intercept, the residue functions would have very different features, leading to a breakdown of factorization (see below).

Diagonal diffraction, i.e., elastic scattering, with the forward amplitude related via the unitarity relation to the total cross section, in terms of the optical analogy can be treated as a shadow of inelastic processes. The stronger are inelastic interactions, the larger is the elastic cross section. The maximum is reached at the unitarity bound, so called "black-disk" limit.

The off-diagonal diffractive dynamics is more involved. Extending the optical analogy, one can interpret the offdiagonal diffractive amplitude as a linear combination of shadows of different inelastic channels, which tend to compensate each others. In the black-disk limit they cancel completely, and diffraction vanishes. These features follow from the quantum-mechanical picture of diffraction [16-18], which can be illustrated by switching to the eigenstate representation [19,20].

As far as a hadron is subject to diffractive excitation, it is apparently not an eigenstate of interaction, but can be expanded over the complete set of eigenstates $|\alpha\rangle$ of the elastic amplitude operator, $\hat{f}_{\mathrm{el}}|\alpha\rangle=f_{\alpha}|\alpha\rangle[3,19,21]$,

$$
|h\rangle=\sum_{\alpha=1} C_{\alpha}^{h}|\alpha\rangle
$$

where the coefficients $C_{\alpha}^{h}$ satisfy the orthogonality relation,

$$
\left\langle h^{\prime} \mid h\right\rangle=\sum_{\alpha=1}\left(C_{\alpha}^{h^{\prime}}\right)^{*} C_{\alpha}^{h}=\delta_{h h^{\prime}}
$$

Correspondingly, the elastic and single diffraction hadronic amplitudes can be expressed via the eigenamplitudes as,

$$
\begin{gathered}
f_{\mathrm{el}}^{h \rightarrow h}=\sum_{\alpha=1}\left|C_{\alpha}^{h}\right|^{2} f_{\alpha} \\
f_{s d}^{h \rightarrow h^{\prime}}=\sum_{\alpha=1}\left(C_{\alpha}^{h^{\prime}}\right)^{*} C_{\alpha}^{h} f_{\alpha}
\end{gathered}
$$

At the unitarity bound, all the eigenamplitudes $\operatorname{Im} f_{\alpha}=1$, so the positively defined elastic amplitude (1.3), as mentioned above, reaches a maximum. At the same time, the off-diagonal diffractive amplitude (1.4) consists of terms with alternating signs, which tend to cancel each other, and the amplitude vanishes in the black-disk limit, according to the orthogonality relation (1.2) [16-18].

Frequently, the failure of the predictions based on factorization, is explained and attempted to be improved by introducing a suppression factor, so called gap survival probability, evaluated within probabilistic models [22,23].
Such an ad hoc way to cure the factorization prescription cannot replace the quantum-mechanical expression (1.4), so it cannot be correct. The diffractive amplitude (1.4), is a linear combination of elastic amplitudes, which contain a rapidity gap by definition. Therefore, this expression does not need any gap survival factor.

\section{B. Dipole representation}

The eigenstates of interaction $|\alpha\rangle$ in high-energy QCD are color dipoles [20]. The eigen amplitudes $f_{\alpha}$ cannot be calculated reliably, but can be extracted from low- $x$ DIS data. Relying on such a color-dipole phenomenology we calculate below the diffractive amplitude (1.4) for dijet production. This process at the Tevatron $p \bar{p} \rightarrow \bar{p}+$ gap + $j j+X$ is characterized by the presence of two jets in the final state, a large rapidity gap void of particles, and a leading anti-proton $\bar{p}$, which survives the collision and remains intact.

The breakdown of diffractive factorization, the most striking result of Ref. [5], was seen as an order of magnitude suppression of the measured dijet diffractive cross section compared to the theoretical predictions based upon the diffractive parton densities fitted to HERA data on diffractive DIS. The main source of this problem, as demonstrated above, is application of the results of the analysis of data on diagonal DIS diffraction to the essentially off-diagonal diffractive excitation of hadrons.

Diffractive gluon Bremsstrahlung off a projectile quark has been studied in the color dipole approach in the limit of small gluon fractional light-cone momentum $\alpha \ll 1$ in Ref. [21]. In the hadronic case diffractive gluon Bremsstrahlung appears to be the leading-twist process due to interaction with the spectator partons [13], that is similar to the Abelian case (see, e.g., Refs. $[9,10]$ ). While for the forward scattering the corresponding process does not vanish (contrary to the Abelian case), QCD factorization is still expected to be broken due to an interplay between hard and soft fluctuations. In this paper, being motivated by the Tevatron data on SD production of dijets, we extend the dipole formalism of Ref. [21] to the case of arbitrary $\alpha$ of diffractively produced gluon, then we apply it for the hadronic case where large distances are necessarily involved and present the key features of the SD-to-inclusive ratio that indicate the dramatic breakdown of diffractive factorization in non-Abelian diffraction. The light-cone dipole approach enables to incorporate such effects coherently at the amplitude level, which has been previously proven to work well in the diffractive Abelian radiation processes [10-12] and diffractive heavy flavor production [13]. In this paper, following the original studies of inclusive $[24,25]$ and diffractive diffractive gluon radiation $[13,21,26]$, we apply the light-cone dipole approach to the analysis of inclusive and diffractive gluon radiation beyond QCD factorization. By comparing the dipole model results with the Tevatron data for the SD-to-inclusive ratio, we 

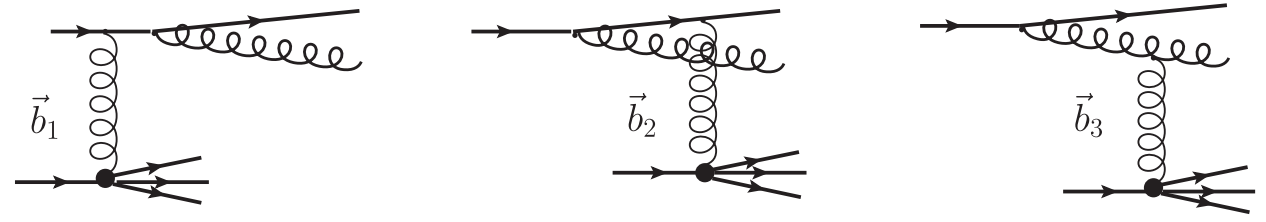

FIG. 1. The leading-order contributions to the gluon Bremsstrahlung mechanism of high- $p_{T}$ back-to-back dijets production in quarknucleon $q N \rightarrow q G X$ scattering.

check whether the gap survival effects are properly accounted for in the dipole treatment of the diffractive non-Abelian radiation.

The paper is organized as follows. In Sec. II, we develop the dipole model formulation of the inclusive dijet production in the target rest frame based upon the gluon Bremsstrahlung mechanism (quark excitation) as well as from the gluon splitting mechanism (gluon excitation). In Sec. III, the models for the universal dipole cross section are briefly discussed in the soft and hard dipole scattering regimes. In Sec. IV, we extend the dipole formulation to the SD dijet production and derive the corresponding partonand hadron-level amplitudes as well as the SD cross sections in the hard scattering limit. Then, in Sec. V we construct the SD-to-inclusive ratio of the cross sections taking into account the CDF Run II experimental constraints on the phase space and present the numerical results. Finally, concluding remarks are given in Sec. VI.

\section{INCLUSIVE BACK-TO-BACK DIJETS}

\section{A. Dijets from quark excitations}

At forward rapidities inclusive production of high- $p_{T}$ jets in the dipole picture is dominated by the gluon Bremsstrahlung mechanism off a projectile quark [24] (similar to the Drell-Yan process [27-30]). The leading order ("skeleton") diagrams of this process are depicted in Fig. 1 . In this case, $x_{1} \equiv p^{+} / P_{1}^{+} \lesssim 1, x_{2} \equiv p^{-} / P_{2}^{-} \ll 1$, where $p$ is the 4-momentum of the radiated gluon, and $P_{1,2}$ are the 4-momenta of the projectile and target nucleons, respectively.

Let us denote the transverse momenta (relative to the projectile quark) of the final quark and gluon as $\vec{p}_{2}$ and $\vec{p}$, respectively, their total momentum as $\overrightarrow{q_{\perp}}=\vec{p}_{2}+\vec{p}$, and the relative momentum as $\vec{\kappa}=\alpha \vec{p}_{2}-\bar{\alpha} \vec{p}$ in terms of the light-cone momentum fraction $\alpha$ carried by the gluon. In the case of collinear projectile quark, the transverse momentum transfer is equal to $\overrightarrow{q_{\perp}}$. Then, the inclusive dijet production amplitude $\hat{B}_{l}\left(\vec{q}_{\perp}, \vec{\kappa}\right)$ reads

$\hat{B}_{l}\left(\vec{q}_{\perp}, \vec{\kappa}\right)=\int d^{2} b d^{2} r e^{i \vec{b} \vec{q}_{\perp}} e^{i \vec{r} \vec{\kappa}} \hat{A}_{l}\left(q N \rightarrow q G N_{8}^{*}\right)$,

in terms of the corresponding amplitude $\hat{A}_{l}\left(q N \rightarrow q G N_{8}^{*}\right)$ found in impact parameter representation as sum over three contributions in Fig. 1

$$
\begin{aligned}
& \hat{A}_{l}\left(q N \rightarrow q G N_{8}^{*}\right) \\
& =\frac{\sqrt{3}}{2} \sum_{a}\left[\tau_{l} \tau_{a}\left\langle N_{8}^{*}\left|\hat{\gamma}_{a}\left(\vec{b}_{1}\right)\right| N\right\rangle-\tau_{a} \tau_{l}\left\langle N_{8}^{*}\left|\hat{\gamma}_{a}\left(\vec{b}_{2}\right)\right| N\right\rangle\right. \\
& \left.\quad-\sum_{b} i f_{l a b} \tau_{b}\left\langle N_{8}^{*}\left|\hat{\gamma}_{a}\left(\vec{b}_{3}\right)\right| N\right\rangle\right] \Psi_{q \rightarrow q G}(\vec{r}, \alpha),
\end{aligned}
$$

where $l$ is the color index of the radiated gluon $G, N_{8}^{*}$ is the color-octet remnant of the target nucleon, for which the completeness relation holds, $\left|N_{8}^{*}\right\rangle\left\langle N_{8}^{*}\right|=1 ; \lambda_{a}=2 \tau_{a}$ are the Gell-Mann matrices; and $\hat{\gamma}_{a}$ is the effective gluonnucleon interaction vertex $G N \rightarrow N_{8}^{*}$. The impact parameters of the projectile, ejectile quarks and the radiated gluon are

$\vec{b}_{1} \equiv \vec{b}, \quad \vec{b}_{2} \equiv \vec{b}-\alpha \vec{r}, \quad \vec{b}_{3} \equiv \vec{b}+\bar{\alpha} \vec{r}, \quad \bar{\alpha} \equiv 1-\alpha$,

such that $\vec{r}$ is the transverse separation of the $q G$ system, and $\vec{b}$ is the distance between its center of gravity and the target $N$. The light-cone distribution function for the $q G$ Fock state (with transversely polarized gluon) in the projectile quark $\hat{\Psi}_{q \rightarrow q G}$ is given by $[24,27,28]$

$$
\begin{aligned}
\hat{\Psi}_{q \rightarrow q G}(\vec{r}, \alpha)= & \frac{2}{\sqrt{3}} \frac{\sqrt{\alpha_{s}}}{2 \pi} \chi_{f}^{\dagger} \hat{\Gamma} \chi_{i} K_{0}(\tau r), \\
\tau^{2}= & \alpha^{2} m_{q}^{2}+(1-\alpha) m_{G}^{2}, \\
\hat{\Gamma}= & i m_{q} \alpha^{2} \vec{e}_{G} \cdot[\vec{n} \times \vec{\sigma}]+\alpha \vec{e}_{G} \cdot[\vec{\sigma} \times \vec{\nabla}] \\
& -i(2-\alpha) \vec{e}_{G} \cdot \vec{\nabla},
\end{aligned}
$$

where $\alpha_{s}=\alpha_{s}\left(\mu^{2}\right)$ is the QCD coupling constant determined at the hard scale $\mu^{2}, m_{G}\left(m_{q}\right)$ is the effective gluon (quark) mass; $\chi$ is the quark spinor, $\vec{e}_{G}$ is the transverse polarization vector of the radiated gluon; $K_{0}(x)$ is the modified Bessel function of the second kind; and $\vec{\nabla} \equiv \partial / \partial \vec{r}$. The corresponding wave function in momentum representation reads

$$
\begin{aligned}
\hat{\tilde{\Psi}}_{q \rightarrow q G}(\vec{\kappa}, \alpha)= & \frac{2 \sqrt{\alpha_{s}}}{\sqrt{3}} \chi_{f}^{\dagger} \hat{\tilde{\Gamma}} \chi_{i} \frac{1}{\kappa^{2}+\tau^{2}} \\
\hat{\tilde{\Gamma}}= & i m_{q} \alpha^{2} \vec{e}_{G} \cdot[\vec{n} \times \vec{\sigma}]+i \alpha \vec{e}_{G} \cdot[\vec{\sigma} \times \vec{\kappa}] \\
& -(2-\alpha)\left(\vec{e}_{G} \cdot \vec{\kappa}\right),
\end{aligned}
$$


If the gluon is radiated with large transverse momentum, it is likely to turn into a hard jet, due to intensive radiation.

The differential cross section for the inclusive $q N \rightarrow$ $q G X$ process has the form,

$$
\begin{aligned}
\frac{d^{3} \sigma_{\mathrm{incl}}(q N \rightarrow q G X)}{d(\ln \alpha) d^{2} \kappa}= & \frac{1}{3(2 \pi)^{2}} \int \frac{d^{2} q_{\perp}}{(2 \pi)^{2}} \\
& \times \sum_{l} \operatorname{Tr}\left[\hat{B}_{l}^{\dagger}\left(\vec{q}_{\perp}, \vec{\kappa}\right) \hat{B}_{l}\left(\vec{q}_{\perp}, \vec{\kappa}\right)\right],
\end{aligned}
$$

where the numerical prefactor indicates at the averaging over colors of the projectile quark. We employ completeness of the remnant $N_{8}^{*}$ states and average over the target nucleon degrees of freedom as follows,

$$
\left\langle N\left|\hat{\gamma}_{a}\left(\vec{b}_{k}\right) \hat{\gamma}_{a^{\prime}}\left(\vec{b}_{l}\right)\right| N\right\rangle=\frac{3}{4} \delta_{a a^{\prime}} \phi\left(\vec{b}_{k}, \vec{b}_{l}\right), \quad \hat{\gamma}_{a}=\hat{\gamma}_{a}^{\dagger} .
$$

Then integrating over $\vec{q}_{\perp}$ one arrives at the differential cross section expressed in terms of the symmetric partial dipole amplitude $\phi\left(\vec{b}_{k}, \vec{b}_{l}\right)=\phi\left(\vec{b}_{l}, \vec{b}_{k}\right)$,

$$
\begin{aligned}
& \frac{d^{3} \sigma_{\text {incl }}(q N \rightarrow q G X)}{d(\ln \alpha) d^{2} \kappa} \\
& =\frac{1}{(2 \pi)^{2}} \int d^{2} r d^{2} r^{\prime} e^{i\left(\vec{r}-\vec{r}^{\prime}\right) \vec{k}} \sum \hat{\Psi}_{q \rightarrow q G}(\vec{r}, \alpha) \hat{\Psi}_{q \rightarrow q G}^{\dagger}\left(\vec{r}^{\prime}, \alpha\right) \\
& \quad \times \sum_{\mathrm{eff}}^{q \rightarrow q G}\left(\vec{r}, \vec{r}^{\prime}, \alpha\right)
\end{aligned}
$$

Here the distribution function squared (averaged over the projectile quark spins) is given by

$$
\begin{aligned}
\sum \hat{\Psi} \hat{\Psi}^{\dagger} \equiv & \sum_{\lambda_{g}= \pm 1} \frac{1}{2} \sum_{\sigma_{f}, \sigma_{i}} \hat{\Psi}_{q \rightarrow q G}(\vec{r}, \alpha) \hat{\Psi}_{q \rightarrow q G}^{\dagger}\left(\vec{r}^{\prime}, \alpha\right) \\
= & \frac{2 \alpha_{s}}{3 \pi^{2}}\left(m_{q}^{2} \alpha^{4} K_{0}(\tau r) K_{0}\left(\tau r^{\prime}\right)\right. \\
& \left.+\left[1+\bar{\alpha}^{2}\right] \tau^{2} \frac{\vec{r} \cdot \vec{r}^{\prime}}{r r^{\prime}} K_{1}(\tau r) K_{1}\left(\tau r^{\prime}\right)\right),
\end{aligned}
$$

and the effective dipole cross section reads,

$$
\begin{aligned}
& \sum_{\mathrm{eff}}^{q \rightarrow q G}\left(\vec{r}, \vec{r}^{\prime}, \alpha\right) \\
&=\int d^{2} b\left\{\phi\left(\vec{b}_{1}, \vec{b}_{1}^{\prime}\right)+\frac{1}{8} \phi\left(\vec{b}_{1}, \vec{b}_{2}^{\prime}\right)-\frac{9}{8} \phi\left(\vec{b}_{1}, \vec{b}_{3}^{\prime}\right)\right. \\
&+\frac{1}{8} \phi\left(\vec{b}_{2}, \vec{b}_{1}^{\prime}\right)+\phi\left(\vec{b}_{2}, \vec{b}_{2}^{\prime}\right)-\frac{9}{8} \phi\left(\vec{b}_{2}, \vec{b}_{3}^{\prime}\right) \\
&\left.-\frac{9}{8} \phi\left(\vec{b}_{3}, \vec{b}_{1}^{\prime}\right)-\frac{9}{8} \phi\left(\vec{b}_{3}, \vec{b}_{2}^{\prime}\right)+\frac{9}{4} \phi\left(\vec{b}_{3}, \vec{b}_{3}^{\prime}\right)\right\} .
\end{aligned}
$$

It depends on impact parameters, $\vec{b}_{1}^{\prime}=\vec{b}_{1} \equiv \vec{b}, \quad \vec{b}_{2}^{\prime} \equiv \vec{b}-\alpha \vec{r}^{\prime}, \quad \vec{b}_{3}^{\prime} \equiv \vec{b}+\bar{\alpha} \vec{r}^{\prime}$.

The partial dipole amplitude $\phi\left(\vec{b}_{k}, \vec{b}_{l}\right)$ introduced in Eq. (2.7) is directly related to the universal dipole-nucleon cross section $\sigma_{q \bar{q}}$ as follows (see also Refs. [21,24])

$$
\begin{aligned}
\sigma_{\bar{q} q}\left(\vec{r}_{1}-\vec{r}_{2}\right) \equiv & \int d^{2} b\left[\phi\left(\vec{b}+\vec{r}_{1}, \vec{b}+\vec{r}_{1}\right)\right. \\
& \left.+\phi\left(\vec{b}+\vec{r}_{2}, \vec{b}+\vec{r}_{2}\right)-2 \phi\left(\vec{b}+\vec{r}_{1}, \vec{b}+\vec{r}_{2}\right)\right],
\end{aligned}
$$

so that the $b$-integration in Eq. (2.10) yields

$$
\begin{aligned}
\sum_{\mathrm{eff}}^{q \rightarrow q G}\left(\vec{r}, \vec{r}^{\prime}, \alpha\right)= & \frac{1}{2}\left\{\sigma_{G q \bar{q}}\left(\bar{\alpha} \vec{r}, \bar{\alpha} \vec{r}+\alpha \vec{r}^{\prime}\right)\right. \\
& +\sigma_{G q \bar{q}}\left(\bar{\alpha} \vec{r}^{\prime}, \bar{\alpha} \vec{r}^{\prime}+\alpha \vec{r}\right)-\sigma_{q \bar{q}}\left(\alpha\left(\vec{r}-\vec{r}^{\prime}\right)\right) \\
& \left.-\sigma_{\mathrm{GG}}\left(\bar{\alpha}\left(\vec{r}-\vec{r}^{\prime}\right)\right)\right\} .
\end{aligned}
$$

The gluonic $G G$ dipole cross section [31] and the effective three-body $G q \bar{q}$ dipole cross section [32,33], read,

$$
\begin{aligned}
\sigma_{\mathrm{GG}}(\vec{r}) & =\frac{9}{4} \sigma_{q \bar{q}}(\vec{r}), \\
\sigma_{G q \bar{q}}\left(\vec{r}_{1}, \vec{r}_{2}\right) & =\frac{9}{8}\left(\sigma_{q \bar{q}}\left(\vec{r}_{1}\right)+\sigma_{q \bar{q}}\left(\vec{r}_{2}\right)\right)-\frac{1}{8} \sigma_{q \bar{q}}\left(\vec{r}_{1}-\vec{r}_{2}\right),
\end{aligned}
$$

respectively.

In the collinear approximation for the projectile parton, the inclusive hadronic $N N \rightarrow q G+X$ cross section reads

$\frac{d^{4} \sigma_{\mathrm{incl}}^{\mathrm{NN}}}{d\left(\ln x_{q}\right) d(\ln \alpha) d^{2} \kappa}=Q\left(x_{q}, \mu^{2}\right) \frac{d^{3} \sigma(q N \rightarrow q G+X)}{d(\ln \alpha) d^{2} \kappa}$,

where $x_{q}$ is the fractional light-cone momentum carried by the projectile quark in the parent nucleon, and the projectile quark distribution distribution function is

$$
Q\left(x_{q}, \mu^{2}\right) \equiv x_{q} q\left(x_{q}, \mu^{2}\right)
$$

at the hard scale $\mu^{2}$ being the invariant mass squared of the produced $q G$ (or dijet) system $\mu^{2} \simeq M_{q G}^{2}$.

\section{B. Dijets from gluon excitations}

At central rapidities inclusive high- $p_{T}$ dijet production can acquire large contributions from the gluon-initiated subprocesses $G N \rightarrow q \bar{q} X$ or $G N \rightarrow G G X$, as is shown in Fig. 2 by upper and lower rows, respectively. 

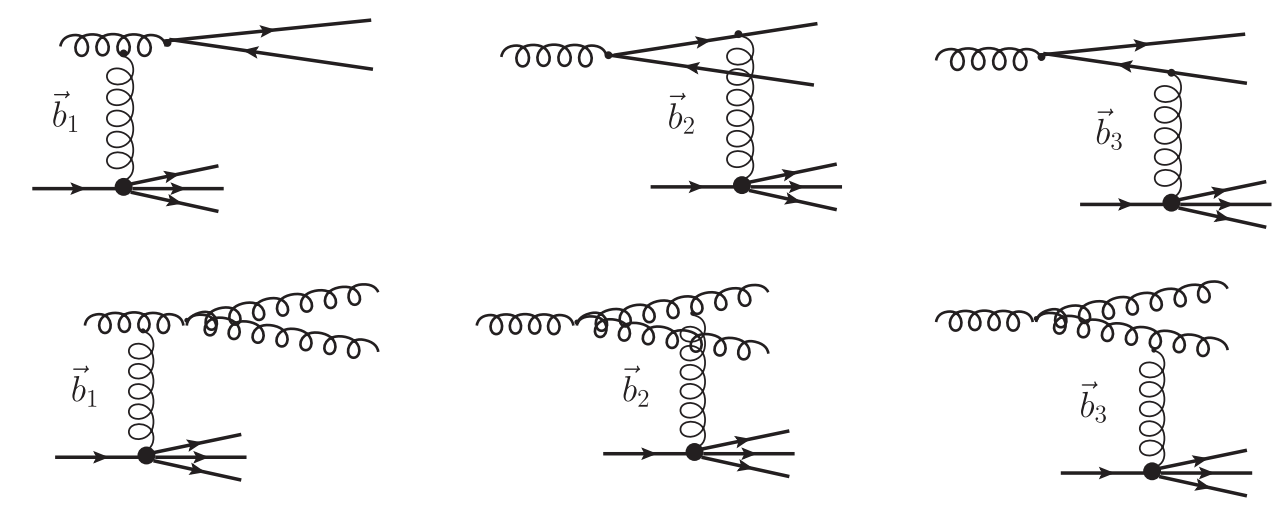

FIG. 2. The leading-order contributions to high- $p_{T}$ dijet production in gluon-nucleon scattering $(G N \rightarrow q \bar{q} X-$ upper row, and $G N \rightarrow G G X$-lower row) in the dipole picture.

The amplitude of the inclusive process $G N \rightarrow q \bar{q} N_{8}^{*}$ is given by the sum of three terms corresponding to the diagrams shown in the upper row of Fig. 2,

$$
\begin{aligned}
& \hat{A}_{l}\left(G N \rightarrow q \bar{q} N_{8}^{*}\right) \\
& =\sqrt{2} \sum_{a} \chi_{q}^{\mu^{\dagger}}\left\{\tau_{a} \tau_{l}\left\langle N_{8}^{*}\left|\hat{\gamma}_{a}\left(\vec{b}_{3}\right)\right| N\right\rangle-\tau_{l} \tau_{a}\left\langle N_{8}^{*}\left|\hat{\gamma}_{a}\left(\vec{b}_{2}\right)\right| N\right\rangle\right. \\
& \left.\quad-i \sum_{c} f_{a l c} \tau_{c}\left\langle N_{8}^{*}\left|\hat{\gamma}_{a}\left(\vec{b}_{1}\right)\right| N\right\rangle\right\} \hat{\Psi}_{G \rightarrow q \bar{q}}(\vec{r}, \alpha) \tilde{\chi}_{\bar{q}}^{\bar{\mu}},
\end{aligned}
$$

where $\tilde{\chi}_{\bar{q}}^{\bar{\mu}}=i \sigma_{y}\left(\chi_{\bar{q}}^{\bar{\mu}}\right)^{*}$, the impact parameters $\vec{b}_{1,2,3}$ are defined in Eq. (2.3), $\chi_{q, \bar{q}}$ are the two-component spinors normalized as

$\sum_{\mu, \bar{\mu}} \tilde{\chi}_{\bar{q}}^{\bar{\mu}}\left(\chi_{q}^{\mu \dagger}\right)^{*}=\hat{1}, \quad \sum_{\mu, \bar{\mu}}\left(\chi_{q}^{\mu \dagger} \hat{a} \tilde{\chi}_{\bar{q}}^{\bar{\mu}}\right)^{*}\left(\chi_{q}^{\mu \dagger} \hat{b} \tilde{\chi}_{\bar{q}}^{\bar{\mu}}\right)=\operatorname{Tr}\left(\hat{a}^{\dagger} \hat{b}\right)$,

and the distribution amplitude of the $G \rightarrow q \bar{q}$ splitting $\hat{\Phi}_{G \rightarrow q \bar{q}}$ reads

$$
\begin{aligned}
\hat{\Psi}_{G \rightarrow q \bar{q}}(\vec{r}, \alpha)= & \frac{\sqrt{\alpha_{s}}}{(2 \pi) \sqrt{2}}\left\{m_{q}(\vec{e} \cdot \vec{\sigma})+i(1-2 \alpha)(\vec{\sigma} \cdot \vec{n})(\vec{e} \cdot \vec{\nabla})\right. \\
& -(\vec{e} \times \vec{n}) \cdot \vec{\nabla}\} K_{0}(\epsilon r),
\end{aligned}
$$

with $\epsilon^{2}=m_{q}^{2}-\alpha \bar{\alpha} m_{G}^{2}$.

When taking square of the total inclusive $G+N \rightarrow$ $q \bar{q}+X$ amplitude

$$
\overline{|A|^{2}}\left(\vec{r}_{1} ; \vec{r}_{2}\right) \equiv \frac{1}{8} \int d^{2} s d\{X\} \sum_{\lambda_{*}, l, \mu, \bar{\mu}}\left\langle A_{l}^{\mu \bar{\mu}}\left(\vec{s}, \vec{r}_{1}\right)\left(A_{l}^{\mu \bar{\mu}}\right)^{\dagger}\left(\vec{s}, \vec{r}_{2}\right)\right\rangle
$$

one performs an averaging over color index and, implicitly, over polarization $\lambda_{*}$ of the projectile gluon $G$ as well as valence quarks and their relative coordinates in the target nucleon. The corresponding inclusive cross section

$$
\begin{aligned}
\frac{d^{3} \sigma_{\text {incl }}(G N \rightarrow q \bar{q} X)}{d(\ln \alpha) d^{2} \kappa}= & \frac{1}{(2 \pi)^{2}} \int d^{2} r d^{2} r^{\prime} e^{i\left(\vec{r}-\vec{r}^{\prime}\right) \vec{\kappa}} \\
& \times \sum \hat{\Psi}_{G \rightarrow q \bar{q}}(\vec{r}, \alpha) \hat{\Psi}_{G \rightarrow q \bar{q}}^{\dagger}\left(\vec{r}^{\prime}, \alpha\right) \\
& \times \sum_{\mathrm{eff}}^{G \rightarrow q \bar{q}}\left(\vec{r}, \vec{r}^{\prime}, \alpha\right)
\end{aligned}
$$

where

$$
\begin{aligned}
& \sum \overline{\Psi_{G \rightarrow q \bar{q}}^{*}}(\alpha, \vec{r}) \hat{\Psi}_{G \rightarrow q \bar{q}}\left(\alpha, \vec{r}^{\prime}\right) \\
& =\frac{\alpha_{s}}{4 \pi^{2}}\left[m_{q}^{2} K_{0}(\epsilon r) K_{0}\left(\epsilon r^{\prime}\right)\right. \\
& \left.\quad+\left(\alpha^{2}+\bar{\alpha}^{2}\right) \epsilon^{2} \frac{\vec{r} \cdot \vec{r}^{\prime}}{r r^{\prime}} K_{1}(\epsilon r) K_{1}\left(\epsilon r^{\prime}\right)\right],
\end{aligned}
$$

and the effective dipole cross section reads

$$
\begin{aligned}
\Sigma_{\mathrm{eff}}^{G \rightarrow q \bar{q}}\left(\vec{r}, \vec{r}^{\prime}, \alpha\right)= & \frac{1}{2}\left\{\sigma_{G q \bar{q}}\left(-\alpha \vec{r}, \bar{\alpha} \vec{r}^{\prime}\right)+\sigma_{G q \bar{q}}\left(\bar{\alpha} \vec{r},-\alpha \vec{r}^{\prime}\right)\right. \\
& \left.-\sigma_{q \bar{q}}\left(\alpha\left(\vec{r}-\vec{r}^{\prime}\right)\right)-\sigma_{q \bar{q}}\left(\bar{\alpha}\left(\vec{r}-\vec{r}^{\prime}\right)\right)\right\},
\end{aligned}
$$

in terms of the $G q \bar{q}$ cross section defined in Eq. (2.14).

Analogically, the amplitude for inclusive $G N \rightarrow$ $G_{1} G_{2} N_{8}^{*}$ process in gluon-target scattering reads [see Fig. 2 (second row)]

$$
\begin{aligned}
& \hat{A}_{l^{\prime} l s}\left(G N \rightarrow G_{1} G_{2} N_{8}^{*}\right) \\
& =\frac{1}{2 \sqrt{6}} \sum_{a, b}\left\{f_{l a b} f_{l^{\prime} s b}\left\langle N_{8}^{*}\left|\hat{\gamma}_{a}\left(\vec{b}_{3}\right)\right| N\right\rangle\right. \\
& \quad-f_{l s b} f_{l^{\prime} a b}\left\langle N_{8}^{*}\left|\hat{\gamma}_{a}\left(\vec{b}_{2}\right)\right| N\right\rangle \\
& \left.\quad+f_{l^{\prime} l b} f_{a s b}\left\langle N_{8}^{*}\left|\hat{\gamma}_{a}\left(\vec{b}_{1}\right)\right| N\right\rangle\right\} \Psi_{G \rightarrow G_{1} G_{2}}(\vec{r}, \alpha),
\end{aligned}
$$


where $s, l^{\prime}, l$ are the color indices of the initial $G$ and final $G_{1}, G_{2}$ gluons having polarizations $\vec{e}, \vec{e}_{1}, \vec{e}_{2}$, respectively, and the $G \rightarrow G_{1} G_{2}$ distribution amplitude is given by

$$
\begin{aligned}
\Psi_{G \rightarrow G_{1} G_{2}}(\vec{r}, \alpha)= & \frac{\sqrt{8 \alpha_{s}}}{\pi}\left\{\alpha \bar{\alpha}\left(\vec{e}_{1}^{*} \cdot \vec{e}_{2}^{*}\right)(\vec{e} \cdot \vec{\nabla})-\alpha\left(\vec{e}_{1}^{*} \cdot \vec{e}\right)\left(\vec{e}_{2}^{*} \cdot \vec{\nabla}\right)\right. \\
& \left.-\bar{\alpha}\left(\vec{e}_{2}^{*} \cdot \vec{e}\right)\left(\vec{e}_{1}^{*} \cdot \vec{\nabla}\right)\right\} K_{0}(\omega r)
\end{aligned}
$$

with $\omega^{2}=m_{G}^{2}(1-\alpha \bar{\alpha})$, such that

$$
\begin{aligned}
& \frac{d^{3} \sigma_{\text {incl }}\left(G N \rightarrow G_{1} G_{2} X\right)}{d(\ln \alpha) d^{2} \kappa} \\
& =\frac{1}{(2 \pi)^{2}} \int d^{2} r d^{2} r^{\prime} e^{i\left(\vec{r}-\vec{r}^{\prime}\right) \vec{\kappa}} \\
& \quad \times \bar{\sum} \Psi_{G \rightarrow G_{1} G_{2}}(\vec{r}, \alpha) \Psi_{G \rightarrow G_{1} G_{2}}^{\dagger}\left(\vec{r}^{\prime}, \alpha\right) \Sigma_{\text {eff }}^{G \rightarrow G_{1} G_{2}}\left(\vec{r}, \vec{r}^{\prime}, \alpha\right)
\end{aligned}
$$

where

$$
\begin{aligned}
\bar{\sum} \Psi_{G \rightarrow G_{1} G_{2}}(\alpha, \vec{r}) \Psi_{G \rightarrow G_{1} G_{2}}^{\dagger}\left(\alpha, \vec{r}^{\prime}\right)= & \frac{8 \alpha_{s} \omega^{2}}{\pi^{2}} \frac{\vec{r} \cdot \vec{r}^{\prime}}{r r^{\prime}}(1-\alpha \bar{\alpha})^{2} \\
& \times K_{1}(\omega r) K_{1}\left(\omega r^{\prime}\right),
\end{aligned}
$$

and the effective dipole cross section reads

$$
\begin{aligned}
\Sigma_{\mathrm{eff}}^{G \rightarrow G_{1} G_{2}}\left(\vec{r}, \vec{r}^{\prime}, \alpha\right)= & \frac{9}{16}\left\{\sigma_{q \bar{q}}(\alpha \vec{r})+\sigma_{q \bar{q}}(\bar{\alpha} \vec{r})\right. \\
& +\sigma_{q \bar{q}}\left(\alpha \vec{r}^{\prime}\right)+\sigma_{q \bar{q}}\left(\bar{\alpha} \vec{r}^{\prime}\right) \\
& +\sigma_{q \bar{q}}\left(\bar{\alpha} \vec{r}+\alpha \vec{r}^{\prime}\right)+\sigma_{q \bar{q}}\left(\alpha \vec{r}+\bar{\alpha} \vec{r}^{\prime}\right) \\
& \left.-2 \sigma_{q \bar{q}}\left(\alpha\left|\vec{r}-\vec{r}^{\prime}\right|\right)-2 \sigma_{q \bar{q}}\left(\bar{\alpha}\left|\vec{r}-\vec{r}^{\prime}\right|\right)\right\} .
\end{aligned}
$$

In the limit of small $\alpha \ll 1$, it can be represented as

$$
\begin{aligned}
& \left.\sum_{\text {eff }}^{G \rightarrow G_{1} G_{2}}\left(\vec{r}, \vec{r}^{\prime}, \alpha\right)\right|_{\alpha \rightarrow 0} \\
& \quad=\frac{1}{2}\left\{\sigma_{3 G}(\vec{r}, \alpha)+\sigma_{3 G}\left(\vec{r}^{\prime}, \alpha\right)-\sigma_{3 G}\left(\vec{r}-\vec{r}^{\prime}, \alpha\right)\right\},
\end{aligned}
$$

in terms of effective 3-gluon cross section

$$
\begin{aligned}
\sigma_{3 G}(\vec{r}, \alpha) & =\frac{9}{8}\left\{\sigma_{q \bar{q}}(\vec{r})+\sigma_{q \bar{q}}(\alpha \vec{r})+\sigma_{q \bar{q}}(\bar{\alpha} \vec{r})\right\} \\
& \simeq \sigma_{\mathrm{GG}}(\vec{r}) \equiv \frac{9}{4} \sigma_{q \bar{q}}(\vec{r}), \quad \alpha \ll 1 .
\end{aligned}
$$

Then for small $\alpha \ll 1$, the ratio between the $q G$ and $G G$ total cross sections

$$
\frac{\sigma_{G \rightarrow G_{1} G_{2}}}{\sigma_{q \rightarrow q G}}=6
$$

is given by the color factors only.

\section{HARD VS SOFT DIPOLE SCATTERING}

The phenomenological dipole cross section is the essential ingredient of the color dipole approach [20]. Typically, it is introduced in the form of a saturated ansatz [34]

$$
\sigma_{q \bar{q}}(x, \vec{r})=\sigma_{0}\left[1-e^{-\frac{r^{2}}{R_{0}^{2}(x)}}\right],
$$

whose Bjorken $x$-dependence is phenomenologically motivated by a wealth of experimental data from HERA. Its parametrization fitted to HERA DIS data known as the Golec-Biernat-Wüsthoff (GBW) model reads

$R_{0}^{2} \equiv \frac{4}{Q_{s}^{2}}, \quad Q_{s}^{2}(x) \equiv Q_{0}^{2}\left(\frac{x_{0}}{x}\right)^{\lambda}, \quad Q_{0}^{2}=1 \mathrm{GeV}^{2}$,

$x_{0}=4.01 \times 10^{-5}, \quad \lambda=0.277, \quad \sigma_{0}=29 \mathrm{mb}$.

Such a parametrization, although does not account for the QCD evolution of the target gluon density, still provides a good overall description of many observables in leptonhadron and hadron-hadron collisions at small $x \lesssim 0.01$ and at not very large $Q^{2}$.

During past two decades, various saturation-based parametrizations for the universal dipole cross section that accommodate QCD evolution has been proposed based upon the observation of Refs. [35-38] that the saturation scale is proportional to the collinear gluon density in the target nucleon

$$
Q_{s}^{2}=Q_{s}^{2}\left(x, \mu^{2}\right) \propto \alpha_{s}\left(\mu^{2}\right) x g\left(x, \mu^{2}\right),
$$

with the hard scale $\mu^{2} \sim 1 / r^{2}$. Provided that this scale is not too large, like in the case under consideration of $p_{T^{-}}$ integrated observables of dijet production, we will not explicitly incorporate such a dependence, but for the sake of simplicity, will employ the GBW parametrization [34].

Besides saturation, a common property of all the dipole parametrization is the color transparency limit [20], meaning that a pointlike colorless object does not interact with external color fields, i.e.,

$$
\sigma_{q \bar{q}}(x, \vec{r}) \simeq \sigma_{0} \frac{r^{2}}{R_{0}^{2}(x)}, \quad r^{2} \ll R_{0}^{2}(x),
$$

which concerns the hard dipole scattering at the scale $\mu \gg Q_{s}(x)$. The quadratic dependence of the universal dipole cross section $\sigma_{q \bar{q}} \propto r^{2}$ is a straightforward consequence of gauge invariance and non-Abelian nature of QCD.

Integrating the inclusive dijet cross section (2.8) over $\vec{\kappa}$, we write,

$\frac{d \sigma_{\text {incl }}(q N \rightarrow q G X)}{d(\ln \alpha)}=\int d^{2} r\left|\Psi_{q \rightarrow q G}(\vec{r}, \alpha)\right|^{2} \Sigma_{\text {eff }}^{q \rightarrow q G}(\vec{r}, \vec{r}, \alpha)$.

Here the effective dipole cross section in the small dipole size limit $r \ll R_{0}\left(x_{2}\right)$ 


$$
\begin{aligned}
& \sum_{\text {eff }}^{q \rightarrow q G}(\vec{r}, \vec{r}, \alpha) \simeq \mathcal{K}_{\text {incl }}^{q \rightarrow q G}\left(x_{2}, \alpha\right) r^{2}, \\
& \mathcal{K}_{\text {incl }}^{q \rightarrow q G}\left(x_{2}, \alpha\right)=\frac{\sigma_{0}}{R_{0}^{2}\left(x_{2}\right)} \cdot\left[\frac{9}{4} \bar{\alpha}+\alpha^{2}\right], \quad x_{2}=\frac{M^{2}}{x_{q} s},
\end{aligned}
$$

and $s$ is the nucleon-nucleon c.m. energy squared. The fully differential cross section for the inclusive $q+G$ production in this approximation takes a very simple form

$$
\begin{aligned}
\frac{d \sigma_{\text {incl }}^{\mathrm{NN}}}{d \Omega} \simeq & \frac{\mathcal{K}_{\text {incl }}^{q \rightarrow q G}\left(x_{2}, \alpha\right)}{(2 \pi)^{2}} q\left(x_{q}, \mu^{2}\right) \int d^{2} r d^{2} r^{\prime} e^{i \vec{k}\left(\vec{r}-\vec{r}^{\prime}\right)}\left(\vec{r} \cdot \vec{r}^{\prime}\right) \\
& \times \Psi_{q \rightarrow q G}(\vec{r}, \alpha) \Psi_{q \rightarrow q G}^{\dagger}\left(\vec{r}^{\prime}, \alpha\right),
\end{aligned}
$$

where the phase space volume element is

$$
d \Omega=d x_{q} d \ln \alpha d^{2} \kappa .
$$

For the gluon-initiated processes $G \rightarrow q \bar{q}$ and $G \rightarrow G_{1} G_{2}$ we have

$$
\begin{aligned}
\mathcal{K}_{\text {incl }}^{G \rightarrow q \bar{q}}\left(x_{2}, \alpha\right) & =\frac{\sigma_{0}}{R_{0}^{2}\left(x_{2}\right)} \cdot\left[1-\frac{9}{4} \alpha \bar{\alpha}\right], \\
\mathcal{K}_{\text {incl }}^{G \rightarrow G_{1} G_{2}}\left(x_{2}, \alpha\right) & =\frac{9 \sigma_{0}}{4 R_{0}^{2}\left(x_{2}\right)} \cdot[1-\alpha \bar{\alpha}],
\end{aligned}
$$

respectively.

In the soft limit $Q^{2} \rightarrow \Lambda_{\mathrm{QCD}}^{2}$ one can reach very small values of $x$ defined in Eq. (3.6) even at low energies. This signals about inappropriate use of variable $x_{2}$ in this limit. In soft and semi-soft reactions such as pion-proton scattering, or diffractive processes Drell-Yan and gluon radiation, the saturation scale depends on the gluon-target collision c.m. energy squared $\hat{s}=x_{q} s$ which is a more appropriate variable than the Bjorken $x$. Such reactions are characterized by the associated scale $Q^{2} \sim \Lambda_{\mathrm{QCD}}^{2} \sim 1 / R_{\text {had }}^{2}$ at the soft hadronic scale $R_{\text {had }}$. Keeping the saturated ansatz of the dipole cross section (3.1), the corresponding parametrization for $\sigma_{0} \rightarrow \bar{\sigma}_{0}(\hat{s})$ and $R_{0} \rightarrow \bar{R}_{0}(\hat{s})$ has been found in Ref. [21]

$$
\begin{aligned}
& \bar{R}_{0}(\hat{s})=0.88 \mathrm{fm}\left(s_{0} / \hat{s}\right)^{0.14}, \\
& \bar{\sigma}_{0}(\hat{s})=\sigma_{\text {tot }}^{\pi p}(\hat{s})\left(1+\frac{3 \bar{R}_{0}^{2}(\hat{s})}{8\left\langle r_{\mathrm{ch}}^{2}\right\rangle_{\pi}}\right) .
\end{aligned}
$$

in terms of the pion-proton total cross section given by $\sigma_{\text {tot }}^{\pi p}(\hat{s})=23.6\left(\hat{s} / s_{0}\right)^{0.08} \mathrm{mb}$ [39], $s_{0}=1000 \mathrm{GeV}^{2}$, the mean pion charge radius squared $\left\langle r_{\mathrm{ch}}^{2}\right\rangle_{\pi}=0.44 \mathrm{fm}^{2}$ [40]. This parametrization describes well the HERA data for the proton structure function at medium-high scales up to $Q^{2} \sim 10 \mathrm{GeV}^{2}$. The model (3.10) will be referred below to as the Kopeliovich-Schäfer-Tarasov model and used in our analysis of diffractive dijet production in high-energy hadronic collisions.

\section{SINGLE-DIFFRACTIVE DIJETS PRODUCTION}

The main contribution to the diffractive dijets production cross section at very forward rapidities is given by the diffractive gluon bremsstrahlung off the projectile valence quarks $q \rightarrow q G$ as is demonstrated in Fig. 3 (for an analogous discussion in the case of diffractive Abelian bremsstrahlung, see Refs. [9-12,14]). At hadron colliders such as Tevatron, however, the jet rapidities may extend down to central values where the contribution from diffractive gluon excitation, given by the gluon splitting subprocesses $G \rightarrow q \bar{q}$ and $G \rightarrow G G$, become important. Diffractive excitation of the projectile sea-quarks also contributes, but negligibly less compared with gluons. In what follows, we discuss all these reactions on the same footing and derive the corresponding SD cross sections.

\section{A. Diffractive excitation of a projectile quark}

The hadron-level SD amplitude with the gluon bremsstrahlung process $q \rightarrow q G$ can be conveniently decomposed into three parts,

$\hat{A}_{l}^{q_{i}, \mathrm{SD}}=\hat{A}_{l}^{q_{i},(\mathrm{I})}+\hat{A}_{l}^{q_{i},(\mathrm{II})}+\hat{A}_{l}^{q_{i},(\mathrm{III})}, \quad \hat{A}_{l}^{\mathrm{SD}} \equiv \sum_{i=1}^{3} \hat{A}_{l}^{q_{i}, \mathrm{SD}}$,

with color index $l$ of the gluon by the projectile quark $q_{i}$ $(i=1,2,3)$. In what follows, we keep the earlier introduced notation notation for the total transverse momentum transfer $\vec{q}_{\perp}$ conjugated to the impact parameter $\vec{b}$, like in the inclusive case.

First, let us consider the three graphs in the upper row of Fig. 3 corresponding to the amplitude $\hat{A}_{l}^{q,(\mathrm{I})}$ of diffractive gluon radiation in nucleon-nucleon scattering $N N \rightarrow$ $(3 q)_{8} G N$. One of the $t$-channel gluons, which couples to the hard scale $\mu^{2}$ (large filled circle in Fig. 3), we call "active" gluon. In order to keep the whole $t$-channel exchange colorless, as is required in a diffractive process, there should be an additional effective color octet exchange between any projectile or produced parton and the target, which we call "screening" gluon. Then, the amplitude $\hat{A}_{l}^{q,(\mathrm{I})}$ is related to the amplitude of diffractive gluon radiation in the quark-nucleon $q N \rightarrow q G N$ scattering $\hat{A}_{l}^{q}$ as follows,

$$
\hat{A}_{l}^{q,(\mathrm{I})}=-2 i\left\langle(3 q)_{8}\left|\hat{A}_{l}^{q}\right|(3 q)_{1}\right\rangle \Phi_{N \rightarrow(3 q)_{1}},
$$

where $\Phi_{N \rightarrow(3 q)_{1}}\left(\vec{r}_{1}, \vec{r}_{2}, \vec{r}_{3} ; \alpha_{1}, \alpha_{2}, \alpha_{3}\right)$ is the nucleon wave function describing a fluctuation of the projectile nucleon into a colorless system $(3 q)_{1}$ of three valence quarks $i=1$, 2, 3 with transverse positions and light-cone momentum fractions $\left\{\vec{r}_{i}\right\}$ and $\left\{\alpha_{i}\right\}$ respectively.

At high energies, the diffractive amplitude is predominantly imaginary. So, using the generalized optical theorem 


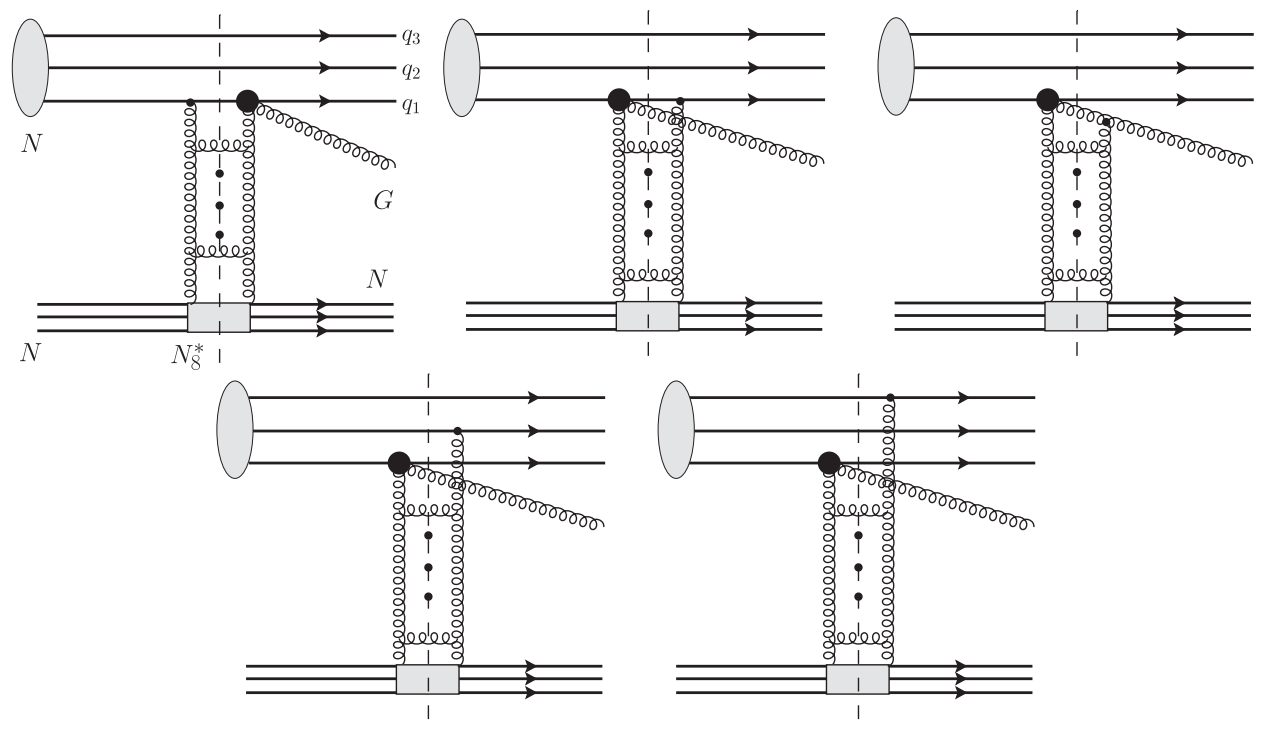

FIG. 3. Dijet production from diffractive quark excitation in $N N$ collisions. Additional graphs come from $q_{1} \leftrightarrow q_{2}$ and $q_{1} \leftrightarrow q_{3}$ permutations. Large filled circle corresponds to three perturbative leading-order contributions depicted in Fig. 1.

for the unitarity cut (dashed vertical lines in Fig. 3) between the "active" and "screening" gluons, and summing up the corresponding contributions, we obtain

$$
\begin{aligned}
\hat{A}_{l}^{q}= & \frac{i}{2} \sum_{N_{8}^{*}}\left[\hat{A}_{l}^{\dagger}\left(q G N \rightarrow q N_{8}^{*}\right) \hat{A}\left(q N \rightarrow q N_{8}^{*}\right)\right. \\
& \left.+\sum_{l^{\prime}} \hat{A}_{l l^{\prime}}^{\dagger}\left(q G N \rightarrow q G^{\prime} N_{8}^{*}\right) \hat{A}_{l^{\prime}}\left(q N \rightarrow q G^{\prime} N_{8}^{*}\right)\right] .
\end{aligned}
$$

Here the first term corresponds to the first diagram in Fig. 3, and the second one to the sum of the second and third diagrams, with explicit summation over intermediate color index of the $G^{\prime}$ gluon $l^{\prime}$ and nucleon octet-charged remnant $N_{8}^{*} \equiv(3 q)_{8}$.

In the impact parameter representation, the scattering amplitude $\hat{A}_{l^{\prime}}\left(q N \rightarrow q G^{\prime} N_{8}^{*}\right)$ has the same form as the inclusive production amplitude Eq. (2.2), while the other amplitudes read

$$
\begin{aligned}
\hat{A}\left(q N \rightarrow q N_{8}^{*}\right)= & \sum_{a} \tau_{a}\left\langle N_{8}^{*}\left|\hat{\gamma}_{a}\left(\vec{b}_{1}\right)\right| N\right\rangle, \\
\hat{A}_{l l^{\prime}}^{\dagger}\left(q G N \rightarrow q G^{\prime} N_{8}^{*}\right)= & \delta_{l l^{\prime}} \sum_{a} \tau_{a}\left\langle N_{8}^{*}\left|\hat{\gamma}_{a}\left(\vec{b}_{2}\right)\right| N\right\rangle-\sum_{a} i f_{l l^{\prime} a}\left\langle N_{8}^{*}\left|\hat{\gamma}_{a}\left(\vec{b}_{3}\right)\right| N\right\rangle, \\
\hat{A}_{l}^{\dagger}\left(q G N \rightarrow q N_{8}^{*}\right)= & \frac{\sqrt{3}}{2} \sum_{a}\left[\tau_{l} \tau_{a}\left\langle N_{8}^{*}\left|\hat{\gamma}_{a}\left(\vec{b}_{1}\right)\right| N\right\rangle-\tau_{a} \tau_{l}\left\langle N_{8}^{*}\left|\hat{\gamma}_{a}\left(\vec{b}_{2}\right)\right| N\right\rangle\right. \\
& \left.-\sum_{b} i f_{l a b} \tau_{b}\left\langle N_{8}^{*}\left|\hat{\gamma}_{a}\left(\vec{b}_{3}\right)\right| N\right\rangle\right] \Psi_{q \rightarrow q G}(\vec{r}, \alpha) .
\end{aligned}
$$

Then, using Eq. (2.7) one arrives at the SD amplitude of $q N \rightarrow q G N$ process

$$
\begin{aligned}
\hat{A}_{l}^{q}= & \frac{3 i \sqrt{3}}{16} \tau_{l} \Psi_{q \rightarrow q G}(\vec{r}, \alpha)\left\{\frac{4}{3}\left(\phi\left(\vec{b}_{1}, \vec{b}_{1}\right)-\phi\left(\vec{b}_{2}, \vec{b}_{2}\right)\right)\right. \\
& \left.+3\left(\phi\left(\vec{b}_{2}, \vec{b}_{3}\right)-\phi\left(\vec{b}_{3}, \vec{b}_{3}\right)\right)\right\},
\end{aligned}
$$

which is infrared finite and vanishes in the color transparency limit $\vec{r} \rightarrow 0$, despite the divergency in the amplitude $\phi\left(\vec{b}_{k}, \vec{b}_{l}\right)$. The symmetry properties of $\phi\left(\vec{b}_{k}, \vec{b}_{l}\right)$ in particular imply, $\sqrt{d^{2} b \sum_{i} \mathcal{C}_{i} \phi\left(\vec{d}_{i}, \vec{d}_{i}\right)=0 \quad \text { for } \sum_{i} \mathcal{C}_{i}=0, \quad \vec{d}_{i}=\vec{b}+\vec{y}_{i}}$

for any $\vec{y}_{i}$ such that in the forward diffractive scattering limit $\vec{q}_{\perp} \rightarrow 0$ we finally have,

$$
\begin{aligned}
\left.\hat{A}_{l}^{q}\left(\vec{q}_{\perp}, \vec{k}\right)\right|_{q_{\perp} \rightarrow 0} & =\int d^{2} b d^{2} r e^{i \vec{\kappa} \vec{r}} \hat{A}_{l}^{q}(\vec{b}, \vec{r}) \\
& =-\frac{9 i \sqrt{3}}{32} \tau_{l} \int d^{2} r e^{i \vec{\kappa} \vec{r}} \Psi_{q \rightarrow q G}(\vec{r}, \alpha) \sigma_{q \bar{q}}(\vec{r}) .
\end{aligned}
$$


For small dipoles $r^{2} \ll R_{0}^{2}(x)$, the diffractive amplitude transforms to

$\left.\hat{A}_{l}^{q}\left(\vec{q}_{\perp}, \vec{\kappa}\right)\right|_{q_{\perp} \rightarrow 0} \simeq \frac{9 i \sqrt{3}}{32} \frac{\sigma_{0}}{R_{0}^{2}(x)} \tau_{l}\left(\vec{\nabla}_{\kappa} \cdot \vec{\nabla}_{\kappa}\right) \hat{\tilde{\Psi}}_{q \rightarrow q G}(\vec{\kappa}, \alpha)$,

where $\vec{\nabla}_{\kappa}=\partial / \partial \vec{\kappa}$, such that

$$
\begin{aligned}
\left(\vec{\nabla}_{\kappa} \cdot \vec{\nabla}_{\kappa}\right) \hat{\tilde{\Psi}}_{q \rightarrow q G}(\vec{\kappa}, \alpha) \simeq & \frac{8 \sqrt{\alpha_{s}}}{\sqrt{3}} \chi_{f}^{\dagger}\left\{i \alpha \vec{e}_{G} \cdot[\vec{\sigma} \times \vec{\kappa}]\right. \\
& \left.-(2-\alpha)\left(\vec{e}_{G} \cdot \vec{\kappa}\right)\right\} \chi_{i} \frac{1}{\kappa^{4}} .
\end{aligned}
$$

Diffractive quark-to-dijet excitation offers another test of factorization. Indeed, in this case there are no spectator partons, which would cause a suppressive gap survival probability, which is usually identified as the reason for factorization breaking. However, factorization fails even without such a gap survival factor. Indeed, the corresponding differential cross section of the SD dijets production in the quark-nucleon scattering $q N \rightarrow q G N$ has the following form (cf. Ref. [21])

$$
\begin{aligned}
& \left.\frac{d^{3} \sigma_{\mathrm{SD}}(q N \rightarrow q G N)}{d(\ln \alpha) d^{2} q_{\perp}}\right|_{q_{\perp} \rightarrow 0} \\
& =\left.\frac{1}{3} \frac{1}{(2 \pi)^{2}} \int \frac{d^{2} \kappa}{(2 \pi)^{2}} \sum_{l} \operatorname{Tr}\left[\hat{A}_{l}^{q}\left(\vec{q}_{\perp}, \vec{\kappa}\right) \hat{A}_{l}^{q \dagger}\left(\vec{q}_{\perp}, \vec{\kappa}\right)\right]\right|_{q_{\perp} \rightarrow 0} \\
& =\frac{1}{16 \pi^{2}} \int d^{2} r\left|\Psi_{q \rightarrow q G}(\vec{r}, \alpha) \tilde{\sigma}_{q \bar{q}}(\vec{r})\right|^{2}, \quad \tilde{\sigma}_{q \bar{q}}(\vec{r}) \equiv \frac{9}{8} \sigma_{q \bar{q}}(\vec{r}),
\end{aligned}
$$

where the factor $1 / 3$ stands for averaging over colors of the projectile quark. In the color transparency (or large radiated gluon transverse momentum) limit, we get

$$
\begin{aligned}
\left.\frac{d^{3} \sigma_{\mathrm{SD}}(q N \rightarrow q G N)}{d(\ln \alpha) d^{2} \kappa d^{2} q_{\perp}}\right|_{q_{\perp} \rightarrow 0}= & \frac{1}{16 \pi^{2}} \frac{81}{64} \frac{\sigma_{0}^{2}}{R_{0}^{4}(x)} \frac{1}{(2 \pi)^{2}} \frac{1}{2} \\
& \times \sum_{i, f, \lambda_{G}}\left|\left(\vec{\nabla}_{\kappa} \cdot \vec{\nabla}_{\kappa}\right) \hat{\tilde{\Psi}}_{q \rightarrow q G}(\vec{\kappa}, \alpha)\right|^{2},
\end{aligned}
$$

where the amplitude squared (averaged over the incoming quark helicities) reads explicitly

$$
\frac{1}{2} \sum_{i, f, \lambda_{G}}\left|\left(\vec{\nabla}_{\kappa} \cdot \vec{\nabla}_{\kappa}\right) \hat{\tilde{\Psi}}_{q \rightarrow q G}(\vec{\kappa}, \alpha)\right|^{2}=\frac{128 \alpha_{s}}{3} \frac{2-\alpha(2-\alpha)}{\kappa^{6}} .
$$

If factorization were true, the diffractive structure functions are nearly scale independent (only logarithmically). Therefore, all the dependence on $\kappa$ comes from the hard parton-parton scattering, i.e., should scale as $1 / \kappa^{4}$, in apparent contradiction with the result (4.10).

Coming to the hadron-level SD amplitude $N N \rightarrow$ $(3 q)_{8} G N$, we define the impact parameters for a gluon radiation off the $i$ th projectile quark $(i=1,2,3)$ in terms of its transverse position $\vec{r}_{i}$ relative to the impact parameter $\vec{b}$ as follows,

$$
\begin{aligned}
& \vec{b}_{1}^{(i)} \equiv \vec{b}+\vec{r}_{i}, \quad \vec{b}_{2}^{(i)} \equiv \vec{b}+\vec{r}_{i}-\alpha \vec{\rho}_{i}, \\
& \vec{b}_{3}^{(i)} \equiv \vec{b}+\vec{r}_{i}+\bar{\alpha} \vec{\rho}_{i}, \quad \vec{\rho}_{i}=\vec{\rho}-\vec{r}_{i},
\end{aligned}
$$

where the difference between transverse coordinates of the radiated gluon, $\vec{\rho}$, and the position of the parent projectile quark is $\vec{r}_{i}$. Thus, the first term in Eq. (4.1) for gluon radiation off the projectile quark $q_{1}$ can be presented as,

$$
\begin{aligned}
\hat{A}_{l}^{q_{1},(\mathrm{I})}= & 2 i \cdot \frac{i \sqrt{3}}{4}\left\langle(3 q)_{8}\left|\tau_{l}^{\left(q_{1}\right)}\right|(3 q)_{1}\right\rangle \Phi_{N \rightarrow(3 q)_{1}} \\
& \times \Psi_{q \rightarrow q G}\left(\vec{\rho}_{1}, \alpha\right) \tilde{\sigma}_{q \bar{q}}\left(\vec{\rho}_{1}\right) \\
= & \frac{i f_{l a b}}{\sqrt{3}}\left\langle(3 q)_{8}\left|\tau_{a}^{\left(q_{1}\right)} \tau_{b}^{\left(q_{1}\right)}\right|(3 q)_{1}\right\rangle \Phi_{N \rightarrow(3 q)_{1}} \\
& \times \Psi_{q \rightarrow q G}\left(\vec{\rho}_{1}, \alpha\right) \tilde{\sigma}_{q \bar{q}}\left(\vec{\rho}_{1}\right),
\end{aligned}
$$

Notice that the mean transverse size of the perturbative fluctuation $q \rightarrow q G$ with a high- $p_{T}$ gluon, controlled by the light-cone distribution function $\Psi_{q \rightarrow q G}\left(\vec{\rho}_{i}\right)$ [see Eq. (2.4)], is much smaller than the interquark separation in the nucleon, which is $R_{N} \sim 1 \mathrm{fm}$, i.e.,

$$
\left|\vec{\rho}_{i}\right| \ll\left|\vec{r}_{i j}\right| \sim R_{N}, \quad i \neq j, \quad \vec{r}_{i j} \equiv \vec{r}_{i}-\vec{r}_{j}
$$

For the second and third terms, $\hat{A}_{l}^{q_{1},(\mathrm{II})}$ and $\hat{A}_{l}^{q_{1},(\mathrm{III})}$, in Eq. (4.1) corresponding to the first and second diagrams in the second row of Fig. 3, respectively, we write,

$$
\begin{aligned}
\hat{A}_{l}^{q_{1},(\mathrm{II})}= & \left\langle(3 q)_{8}\left|\hat{A}^{\dagger}\left(q_{2} N \rightarrow q_{2} N_{8}^{*}\right) \hat{A}_{l}\left(q_{1} N \rightarrow q_{1} G N_{8}^{*}\right)\right|(3 q)_{1}\right\rangle \Phi_{N \rightarrow(3 q)_{1}} \\
= & \frac{3 \sqrt{3} \Phi_{N \rightarrow(3 q)_{1}} \Psi_{q \rightarrow q G}\left(\vec{\rho}_{1}, \alpha\right)}{16}\left\{\left\langle(3 q)_{8}\left|\tau_{a}^{\left(q_{2}\right)} \tau_{l}^{\left(q_{1}\right)} \tau_{a}^{\left(q_{1}\right)}\right|(3 q)_{1}\right\rangle\left(\sigma_{q \bar{q}}\left(\vec{r}_{12}\right)-\sigma_{q \bar{q}}\left(\vec{r}_{12}+\bar{\alpha} \vec{\rho}_{1}\right)\right)\right. \\
& \left.+\left\langle(3 q)_{8}\left|\tau_{a}^{\left(q_{2}\right)} \tau_{a}^{\left(q_{1}\right)} \tau_{l}^{\left(q_{1}\right)}\right|(3 q)_{1}\right\rangle\left(\sigma_{q \bar{q}}\left(\vec{r}_{12}+\bar{\alpha} \vec{\rho}_{1}\right)-\sigma_{q \bar{q}}\left(\vec{r}_{12}-\alpha \vec{\rho}_{1}\right)\right)\right\},
\end{aligned}
$$




$$
\begin{aligned}
\hat{A}_{l}^{q_{1},(\mathrm{III})}= & \left\langle(3 q)_{8}\left|\hat{A}^{\dagger}\left(q_{3} N \rightarrow q_{3} N_{8}^{*}\right) \hat{A}_{l}\left(q_{1} N \rightarrow q_{1} G N_{8}^{*}\right)\right|(3 q)_{1}\right\rangle \Phi_{N \rightarrow(3 q)_{1}} \\
= & \frac{3 \sqrt{3} \Phi_{N \rightarrow(3 q)_{1}} \Psi_{q \rightarrow q G}\left(\vec{\rho}_{1}, \alpha\right)}{16}\left\{\left\langle(3 q)_{8}\left|\tau_{a}^{\left(q_{3}\right)} \tau_{l}^{\left(q_{1}\right)} \tau_{a}^{\left(q_{1}\right)}\right|(3 q)_{1}\right\rangle\left(\sigma_{q \bar{q}}\left(\vec{r}_{13}\right)-\sigma_{q \bar{q}}\left(\vec{r}_{13}+\bar{\alpha} \vec{\rho}_{1}\right)\right)\right. \\
& \left.+\left\langle(3 q)_{8}\left|\tau_{a}^{\left(q_{3}\right)} \tau_{a}^{\left(q_{1}\right)} \tau_{l}^{\left(q_{1}\right)}\right|(3 q)_{1}\right\rangle\left(\sigma_{q \bar{q}}\left(\vec{r}_{13}+\bar{\alpha} \vec{\rho}_{1}\right)-\sigma_{q \bar{q}}\left(\vec{r}_{13}-\alpha \vec{\rho}_{1}\right)\right)\right\},
\end{aligned}
$$

in terms of the partial amplitudes given in Eqs. (2.2) and (4.4).

In practical calculations, it is convenient to employ the following relation

$$
\left(\tau_{a}^{\left(q_{1}\right)}+\tau_{a}^{\left(q_{2}\right)}+\tau_{a}^{\left(q_{3}\right)}\right)\left|(3 q)_{1}\right\rangle=0
$$

and a more generic formula for cyclic permutations $\left\{q_{1}, q_{2}, q_{3}\right\}$ of the products of $\tau^{\left(q_{j}\right)}$-matrices,

$\left(P^{q_{1}} P^{q_{2}} P^{q_{3}}+P^{q_{2}} P^{q_{3}} P^{q_{1}}+P^{q_{3}} P^{q_{1}} P^{q_{2}}\right)\left|(3 q)_{1}\right\rangle=0$,

$P^{q_{j}}=\tau_{a}^{q_{j}} \tau_{b}^{q_{j}} \ldots$

for any product of $\tau$-matrices $P^{q_{j}}$ along a quark line $q_{j}$, $j=1,2,3$ or unity. Averaging over the nucleon state $\left|(3 q)_{1}\right\rangle$ in the SD amplitude squared is performed as follows

$$
\begin{aligned}
& \left\langle(3 q)_{1}\left|A\left(\tau^{\left(q_{1}\right)}\right) B\left(\tau^{\left(q_{2}\right)}\right) C\left(\tau^{\left(q_{3}\right)}\right)\right|(3 q)_{1}\right\rangle \\
& =\frac{1}{6}(\operatorname{Tr}[A] \operatorname{Tr}[B] \operatorname{Tr}[C]+\operatorname{Tr}[A B C]+\operatorname{Tr}[A C B] \\
& \quad-\operatorname{Tr}[A] \operatorname{Tr}[B C]-\operatorname{Tr}[B] \operatorname{Tr}[A C]-\operatorname{Tr}[C] \operatorname{Tr}[A B])
\end{aligned}
$$

where $A, B, C$ are any products of $\tau$-matrices corresponding to $q_{1,2,3}$ projectile quarks, respectively.

Assuming the saturated form of the dipole cross section, up to the terms containing the first power of $\rho_{i} \ll r_{i j}$, we write for two distinct cases

$$
\text { hard regime: } \sigma_{q \bar{q}}\left(\vec{\rho}_{i}\right) \simeq \sigma_{0} \frac{\rho_{i}^{2}}{R_{0}^{2}(x)} \text {, }
$$

soft regime: $\sigma_{q \bar{q}}\left(\vec{r}_{i j}\right)-\sigma_{q \bar{q}}\left(\vec{r}_{i j}-\alpha \vec{\rho}_{i}\right)$

$$
\simeq 2 \alpha\left(\vec{\rho}_{i} \cdot \vec{r}_{i j}\right) \frac{\bar{\sigma}_{0}(\hat{s})}{\bar{R}_{0}^{2}(\hat{s})} e^{-r_{i j}^{2} / \bar{R}_{0}^{2}(\hat{s})},
$$

where the sets of parameters in the universal dipole cross section $\left\{\sigma_{0}, R_{0}(x)\right\}$ and $\left\{\bar{\sigma}_{0}(\hat{s}), \bar{R}_{0}(\hat{s})\right\}$ are determined in the hard-dipole scattering [GBW model (3.2)] and softdipole scattering [Kopeliovich-Schäfer-Tarasov model (3.10)] regimes, respectively. Provided that $\rho_{i} \ll r_{i j}$, we can safely neglect the interference terms for gluon emissions off different projectile quarks, such that only the diagonal product,

$$
\begin{aligned}
\left|\Psi_{q \rightarrow q G}\left(\vec{\rho}_{i}, \alpha\right)\right|^{2}= & \frac{4}{3} \frac{\alpha_{s}\left(\mu^{2}\right)}{2 \pi^{2}}\left\{m_{q}^{2} \alpha^{4} K_{0}^{2}\left(\tau \rho_{i}\right)\right. \\
& \left.+\left[1+(1-\alpha)^{2}\right] \tau^{2} K_{1}^{2}\left(\tau \rho_{i}\right)\right\}
\end{aligned}
$$

contributes to the final result for the (integrated) SD cross section.

When computing the SD amplitude squared we have to use the completeness relation $\left|N_{8}^{*}\right\rangle\left\langle N_{8}^{*}\right|=1$ which accounts for the momentum conservation for the nucleon remnant wave function $\Psi_{N_{8}^{*}}$. More explicitly,

$$
\begin{aligned}
& \sum_{N_{8}^{*}} \Psi_{N_{8}^{*}}\left(\vec{r}_{1}, \vec{r}_{2}, \vec{r}_{3} ;\left\{x_{q}^{1,2, \ldots}\right\},\left\{x_{g}^{1,2, \ldots}\right\}\right) \\
& \quad \times \Psi_{N_{8}^{*}}^{*}\left(\vec{r}_{1}^{\prime}, \vec{r}_{2}^{\prime}, \vec{r}_{3}^{\prime} ;\left\{x_{q}^{\prime 1,2, \ldots}\right\},\left\{x_{g}^{\prime 1,2, \ldots}\right\}\right) \\
& =\delta\left(\vec{r}_{1}-\vec{r}_{1}^{\prime}\right) \delta\left(\vec{r}_{2}-\vec{r}_{2}^{\prime}\right) \delta\left(\vec{r}_{3}-\vec{r}_{3}^{\prime}\right) \prod_{j} \delta\left(x_{q / g}^{j}-x_{q / g}^{\prime j}\right) .
\end{aligned}
$$

The wave function of the initial nucleon state $\Phi_{N \rightarrow(3 q)_{1}}$ depends on transverse coordinates and fractional momenta of all the projectile (valence and sea) quarks and gluons. We assume that all sea quarks and gluons are localized within gluonic "spots", around the constituent valence quarks, whose small transverse size $\sim 0.3 \mathrm{fm}$. The smallness of the spots allows to explain the observed weakness of diffractive gluon radiation [21] (the puzzling smallness of the triplePomeron coupling). There are many other observables confirming such a conclusion [41]). This picture supports the popular two-step model [42-44], in which the initial valence-quark distribution function is fixed at a low scale, and then is developed to a higher scale perturbatively by radiative generation of the sea and gluons.

Thus, the valence-like spatial wave function of the proton introduced at a low scale, is not subject to further variations as function of scale. For the impact parameters $r_{1}, r_{2}, r_{3}$ of the valence quarks we use the symmetric (normalized) Gaussian parametrization of the valence part of the proton wave function reads

$$
\begin{aligned}
\left|\Phi_{N \rightarrow(3 q)_{1}}\right|^{2}= & \frac{3 a^{2}}{\pi^{2}} e^{-a\left(r_{1}^{2}+r_{2}^{2}+r_{3}^{2}\right)} \mathcal{R}\left(\left\{x_{q}\right\},\left\{x_{g}\right\}\right) \\
& \times \delta\left(\vec{r}_{1}+\vec{r}_{2}+\vec{r}_{3}\right) \delta\left(1-\sum_{j=1}^{\infty} x_{q}^{j}-\sum_{j=1}^{\infty} x_{g}^{j}\right),
\end{aligned}
$$


where $a \equiv\left\langle r_{\text {ch }}^{2}\right\rangle_{p}^{-1}$ is the inverse proton mean charge radius squared, $\mathcal{R}$ is the generalized parton distribution function in the projectile nucleon. In fact, the spatial distribution of the valence quarks in the proton, even the string configuration (triangle vs star shapes), are still under debate. Different models were tested in Ref. [45] on data of soft diffraction. Only the Model IV with symmetric dependence on $r_{1}, r_{2}$, $r_{3}$, and the saturated dipole cross section, was found to be able to explain the observed puzzling smallness (only few percent of elastic) of the low-mass diffraction cross section. The latter is described by the $\mathbb{P P R}$ term in the triple-Regge phenomenology [46]. It corresponds to diffractive excitation of the valence quark skeleton (in contrast to diffractive gluon radiation, giving the $\mathbb{P P P}$ term), this is why smallmass diffraction is so sensitive to the valence quark distribution.

In the case of diffractive quark excitation we obtain

$$
\begin{aligned}
& \int \prod_{j \neq 1} d x_{q}^{j} \prod_{k} d x_{g}^{k} \delta\left(1-\sum_{j=1}^{\infty} x_{q}^{j}-\sum_{j=1}^{\infty} x_{g}^{j}\right) \mathcal{R}\left(\left\{x_{q}\right\},\left\{x_{g}\right\}\right) \\
& \quad=q\left(x_{q}, \mu^{2}\right)
\end{aligned}
$$

in terms of the quark PDF $q\left(x_{q}, \mu^{2}\right)$ where the projectile (valence or sea) quark momentum fraction is $x_{q}^{1} \equiv x_{q}$.

The SD quark-gluon dijet production cross section in nucleon-nucleon collisions $N+N \rightarrow q G X+N$ is found as

$$
\begin{aligned}
\left.\frac{d^{3} \sigma_{\mathrm{SD}}}{d \ln \alpha d^{2} q_{\perp}}\right|_{q_{\perp} \rightarrow 0}= & \frac{1}{(4 \pi)^{2}} \int d^{2} r_{1} d^{2} r_{2} d^{2} r_{3} \prod_{i, j} d x_{q}^{i} d x_{g}^{j} \\
& \times \int d^{2} \rho \sum \hat{A}_{l}^{\mathrm{SD}}\left(\hat{A}_{l}^{\mathrm{SD}}\right)^{\dagger}
\end{aligned}
$$

where $q_{\perp}^{2}=-t$. The momentum conservation reduces the integral over the incoming nucleon wave function as

$$
\begin{gathered}
\int d^{2} r_{1} d^{2} r_{2} d^{2} r_{3} e^{-a\left(r_{1}^{2}+r_{2}^{2}+r_{3}^{2}\right)} \delta\left(\vec{r}_{1}+\vec{r}_{2}+\vec{r}_{3}\right) \\
=\frac{1}{9} \int d^{2} r_{12} d^{2} r_{13} e^{-\frac{2 a}{3}\left(r_{12}^{2}+r_{13}^{2}+\vec{r}_{12} \cdot \vec{r}_{13}\right)}
\end{gathered}
$$

such that the basic integrals appearing in the SD cross section

$$
\begin{aligned}
& \frac{3 a^{2}}{\pi^{2}} \frac{1}{9} \int d^{2} R_{1} d^{2} R_{2} e^{-\frac{2 a}{3}\left(R_{1}^{2}+R_{2}^{2}+\vec{R}_{1} \cdot \vec{R}_{2}\right)} e^{-\left(R_{i}^{2}+R_{j}^{2}\right) / \bar{R}_{0}^{2}}\left(\vec{R}_{i} \cdot \vec{R}_{j}\right) \\
& \quad i, j=1,2,3
\end{aligned}
$$

where $\vec{R}_{1} \equiv \vec{r}_{12}, \vec{R}_{2} \equiv \vec{r}_{13}, \vec{R}_{3} \equiv \vec{r}_{23}=\vec{r}_{13}-\vec{r}_{12}$, can be taken fully analytically. Finally, as usual the SD cross section is the forward limit is inversely proportional to the standard Regge-parametrized diffractive $t$-slope, $B_{\mathrm{SD}}(s)$, namely,

$$
\begin{aligned}
\frac{d^{2} \sigma_{\mathrm{SD}}}{d \Omega} & \left.\simeq \frac{1}{B_{\mathrm{SD}}(s)} \frac{d^{3} \sigma_{\mathrm{SD}}}{d \Omega d t}\right|_{t \rightarrow 0}, \\
B_{\mathrm{SD}}(s) & \simeq\left\langle r_{\mathrm{ch}}^{2}\right\rangle_{p} / 3+2 \alpha_{\mathbb{P}}^{\prime} \ln \left(s / s_{1}\right), \quad s_{1}=1 \mathrm{GeV}^{2},
\end{aligned}
$$

where $\alpha_{\mathbb{P}}^{\prime}=0.25 \mathrm{GeV}^{-2}$ and the phase space volume element $d \Omega$ is defined in Eq. (3.8).

Following the above footsteps, straightforward calculations lead to the following representation of the fully differential cross section for the SD $q G$ production in nucleon-nucleon collisions

$$
\begin{aligned}
\frac{d \sigma_{\mathrm{SD}}^{q \rightarrow q G}}{d \Omega} \simeq & \frac{\mathcal{K}_{\mathrm{SD}}^{q \rightarrow q G}(s, \hat{s}, \alpha)}{(2 \pi)^{2}} q\left(x_{q}, \mu^{2}\right) \int d^{2} \rho d^{2} \rho^{\prime} e^{i \vec{\kappa}\left(\vec{\rho}-\vec{\rho}^{\prime}\right)}\left(\vec{\rho} \cdot \vec{\rho}^{\prime}\right) \\
& \times \sum \hat{\Psi}_{q \rightarrow q G}(\vec{\rho}, \alpha) \hat{\Psi}_{q \rightarrow q G}^{\dagger}\left(\vec{\rho}^{\prime}, \alpha\right)
\end{aligned}
$$

where

$$
\begin{aligned}
\mathcal{K}_{\mathrm{SD}}^{q \rightarrow q G}= & \frac{1}{B_{\mathrm{SD}}} \frac{9 a \bar{\sigma}_{0}(\hat{s})^{2}}{256 \pi}\left\{\mathcal{W}_{1}(\hat{s})\left[1-\frac{2 \alpha}{3}+\frac{7 \alpha^{2}}{27}\right]\right. \\
& \left.+\mathcal{W}_{2}(\hat{s})\left[1+\frac{2 \alpha}{3}-\frac{13 \alpha^{2}}{27}\right]\right\},
\end{aligned}
$$

where the $\hat{s}$-dependent functions read

$$
\begin{aligned}
\mathcal{W}_{1}(\hat{s}) & =\frac{8}{\left(4+a \bar{R}_{0}^{2}\right)^{2}}+\frac{12}{\left(12+a \bar{R}_{0}^{2}\right)^{2}}, \quad \hat{s}=x_{q} s, \\
\bar{R}_{0} & =\bar{R}_{0}(\hat{s}), \\
\mathcal{W}_{2}(\hat{s}) & =\frac{6 a^{2} \bar{R}_{0}^{4}}{\left(3+8 a \bar{R}_{0}^{2}+a^{2} \bar{R}_{0}^{4}\right)^{2}}-\frac{a^{2} \bar{R}_{0}^{4}}{\left(3+4 a \bar{R}_{0}^{2}+a^{2} \bar{R}_{0}^{4}\right)^{2}} .
\end{aligned}
$$

\section{B. Diffractive excitation of a projectile gluon}

Turning now to the diffractive gluon excitations, the differential SD cross sections can be written as

$$
\begin{aligned}
\frac{d \sigma_{\mathrm{SD}}^{G \rightarrow q \bar{q}}}{d \Omega} \simeq & \frac{\mathcal{K}_{\mathrm{SD}}^{G \rightarrow q \bar{q}}(s, \hat{s}, \alpha)}{(2 \pi)^{2}} g\left(x_{g}, \mu^{2}\right) \int d^{2} \rho d^{2} \rho^{\prime} e^{i \vec{\kappa}\left(\vec{\rho}-\vec{\rho}^{\prime}\right)}\left(\vec{\rho} \cdot \vec{\rho}^{\prime}\right) \\
& \times \sum \hat{\Psi}_{G \rightarrow q \bar{q}}(\vec{\rho}, \alpha) \hat{\Psi}_{G \rightarrow q \bar{q}}^{\dagger}\left(\vec{\rho}^{\prime}, \alpha\right), \quad \hat{s}=x_{g} s \\
\frac{d \sigma_{\mathrm{SD}}^{G \rightarrow G_{1} G_{2}}}{d \Omega} \simeq & \frac{\mathcal{K}_{\mathrm{SD}}^{G \rightarrow G_{1} G_{2}}(s, \hat{s}, \alpha)}{(2 \pi)^{2}} g\left(x_{g}, \mu^{2}\right) \\
& \times \int d^{2} \rho d^{2} \rho^{\prime} e^{i \vec{\kappa}\left(\vec{\rho}-\vec{\rho}^{\prime}\right)}\left(\vec{\rho} \cdot \vec{\rho}^{\prime}\right) \\
& \times \sum \hat{\Psi}_{G \rightarrow G_{1} G_{2}}(\vec{\rho}, \alpha) \hat{\Psi}_{G \rightarrow G_{1} G_{2}}^{\dagger}\left(\vec{\rho}^{\prime}, \alpha\right)
\end{aligned}
$$


for $G \rightarrow q \bar{q}$ and $G \rightarrow G_{1} G_{2}$ subprocesses, respectively. In analogy with the diffractive bremsstrahlung process discussed in detail above, we find

$$
\begin{aligned}
& \mathcal{K}_{\mathrm{SD}}^{G \rightarrow q \bar{q}}=\frac{1}{B_{\mathrm{SD}}} \frac{9 a \bar{\sigma}_{0}(\hat{s})^{2}}{256 \pi}\left\{\mathcal{W}_{1}(\hat{s})\left[\frac{16}{27}-\frac{4 \alpha}{3}+\alpha^{2}\right]\right. \\
&\left.+\mathcal{W}_{2}(\hat{s})\left[\frac{32}{27}-\frac{8 \alpha}{3}+\alpha^{2}\right]\right\}, \\
& \mathcal{K}_{\mathrm{SD}}^{G \rightarrow G_{1} G_{2}}=\frac{1}{B_{\mathrm{SD}}} \frac{9 a \bar{\sigma}_{0}(\hat{s})^{2}}{256 \pi}\left\{\mathcal{W}_{1}(\hat{s})\left[\frac{5}{6}-\alpha \bar{\alpha}\right]\right. \\
&\left.+\mathcal{W}_{2}(\hat{s})\left[\frac{1}{6}-\alpha \bar{\alpha}\right]\right\},
\end{aligned}
$$

where $\mathcal{W}_{1,2}$ are defined above in Eq. (4.30). In what follows, these formulas will be used in analysis of the SD-to-inclusive ratio.

\section{DIFFRACTIVE TO INCLUSIVE RATIO}

The CDF Run II experimental data [47] on SD dijet production are given, in particular, in terms of the SD-toinclusive ratio $\mathcal{R}_{\mathrm{SD} / \text { incl }}$, which is defined as follows

$\mathcal{R}_{\mathrm{SD} / \text { incl }}=\frac{\Delta \sigma_{\mathrm{SD}} / \Delta \xi}{\Delta \sigma_{\text {incl }}}, \quad \Delta \xi=0.06, \quad \xi \equiv 1-x_{F}=\frac{M_{X}^{2}}{s}$,

where $M_{X}$ is the invariant mass squared of the diffractive system $X, M_{X}^{2}$, containing the dijet, $x_{F}$ is the Feynman variable of the recoil antiproton, $\Delta \sigma_{\mathrm{SD}}\left(\Delta \sigma_{\text {incl }}\right)$ are the SD (inclusive) dijet cross sections integrated over the detector acceptance regions in $\xi \equiv 1-x_{F}$ variable, $0.03<\xi<$ 0.09 , in jet pseudorapidities, $\left|\eta_{1,2}\right|<2.5$, in jet transverse energies, $E_{T}^{1,2}>5 \mathrm{GeV}$, and in the antiproton transverse momentum squared, $|t|<1 \mathrm{GeV}^{2}$. The SD-to-inclusive ratio is then measured as function of the hard scale $Q^{2} \gg$ $R_{0}^{2}$ of the dijet and $x_{\mathrm{Bj}}$,

$$
Q^{2}=\frac{\left(E_{T}^{1}+E_{T}^{2}\right)^{2}}{4}, \quad x_{\mathrm{Bj}}=\frac{1}{\sqrt{s}} \sum_{i=1}^{3 \mathrm{jets}} E_{T}^{i} e^{-\eta_{i}} .
$$

It is difficult to make one-to-one correspondence between theory and data for the observables entering Eq. (5.1), but one can rely on approximations. Considering, for example, the gluon Bremsstrahlung mechanism $q \rightarrow q G$ as a suitable example which was thoroughly discussed in the previous sections, a dominant contribution to the sum in Eq. (5.2) comes from the high- $p_{T}$ gluon jet $G$ with a small longitudinal momentum fraction $x_{G} \ll 1$. Indeed, in the high- $p_{T}$ limit, the leading jets are mostly back-to-back, i.e., $p_{T}^{G} \sim p_{T}^{q} \sim E_{T}^{1,2}$, the third subleading jet is more likely to be produced at a smaller transverse momentum $p_{T}^{\text {jet }=3} \ll E_{T}^{1,2}$, while the gluon Bremstrahlung is enhanced at small $\alpha \ll 1$ and thus is radiated at smallest pseudorapidity among the leading jets such that

$$
x_{G}=x_{q} \alpha, \quad x_{G} \ll x_{q}<1 .
$$

Besides, the invariant mass squared of the dijet system, $M^{2}$, can be approximately identified with the hard scale $Q^{2}$, i.e.,

$$
\mu^{2} \simeq M^{2} \simeq Q^{2} .
$$

As we will see below, these approximations are vital for a comparison of the dipole model results with the data.

In the experimental definition (5.1), the numerator

$$
\frac{\Delta \sigma_{\mathrm{SD}}}{\Delta \xi} \sim \frac{d \sigma_{\mathrm{SD}}}{d \xi}
$$

is essentially the differential SD dijet cross section averaged over the bin interval $\Delta \xi$. The dipole formula for the differential SD dijet cross section (4.28) is differential in dijet mass squared $M^{2}=Q^{2}$ (or $x_{2}$ ), and not in $M_{X}^{2}$, so the analysis of its $\xi$ dependence as well as implementation of $\xi$ cuts cannot be directly performed. Following the proposal of Ref. [11], the way out of this issue is to employ the $\xi$-dependence provided by the phenomenological SD cross section in the triple-Regge form [46]

$-\frac{d^{2} \sigma_{\mathrm{SD}}^{\mathrm{pp}}}{d \xi d q_{\perp}^{2}}=\sqrt{\frac{s_{1}}{s}} \frac{G_{\mathbb{P P R R}}(0)}{\xi^{3 / 2}} e^{-B_{\mathbb{P P R}}^{\mathrm{pp}} q_{\perp}^{2}}+\frac{G_{3 \mathbb{P}}(0)}{\xi} e^{-B_{3 \mathrm{P}}^{\mathrm{pp}} q_{\perp}^{2}}$,

such that the main effect of constraints on $\xi$ variable in this Regge-based cross section and in our result (4.28) is expected to be roughly the same. In the above formula (5.6), we use the results of Ref. [46]

$$
\begin{aligned}
s_{1} & =1 \mathrm{GeV}^{2}, \quad B_{\mathbb{P P} i}^{\mathrm{pp}}=R_{\mathbb{P P} i}^{2}-2 \alpha_{\mathbb{P}}^{\prime} \ln \xi, \quad i=\mathbb{P}, \mathbb{R}, \\
G_{3 \mathbb{P}}(0) & =G_{\mathbb{P P R}}(0)=3.2 \mathrm{mb} / \mathrm{GeV}^{2}, \quad R_{3 \mathbb{P}}^{2}=4.2 \mathrm{GeV}^{-2}, \\
R_{\mathbb{P P R}}^{2} & =1.7 \mathrm{GeV}^{-2},
\end{aligned}
$$

where $\alpha_{\mathbb{P}}^{\prime} \approx 0.25 \mathrm{GeV}^{-2}$ is the Pomeron trajectory slope. Although these parameters were determined by the fit to data long time ago at relatively low energies (ISR), they well predicted data on diffraction at LHC (see Appendix A in [48]).

When integrating Eq. (5.6) over $\xi$ interval allowed by the detector constraints, its upper limit is equal to the maximal measured $\xi_{\max }=0.09$ (the largest momentum that can be taken by the "active" gluon) while its minimal value coincides with $x_{\mathrm{Bj}}$ characterizing the hard dijet system. Then, the correction factor relating the integrated SD cross section with the experimentally constrained $\Delta \sigma_{\mathrm{SD}}$ as a function of $x_{\mathrm{Bj}}$ reads 


$$
\delta=\frac{\int d t \int_{x_{\mathrm{Bj}}}^{\xi_{\max }} d \xi \frac{d^{2} \sigma}{d t d \xi}}{\int d t \int_{\xi^{*}}^{0.3} d \xi \frac{d^{2} \sigma}{d t d \xi}}, \quad \xi^{*}=\frac{Q^{2}}{s} \ll x_{\mathrm{Bj}}
$$

where $\xi^{*}$ is associated with the minimal produced diffractive mass $X$, containing only the dijet. As the result is practically nonsensitive to the upper limit of $\xi$, we fix it to 0.3 corresponding to a situation when a constituent quark in the target looses most of its energy into a hard radiation of the $t$-channel gluon [11]. Notably, the correction factor (5.7) automatically accounts for the jet pseudorapidity constraint such that the resulting SD cross section vanishes when approaching the kinematical boundary $x_{\mathrm{Bj}} \rightarrow \xi_{\max }$ as expected.

The most important results of the previous sections, are the dipole formulas for the differential inclusive and SD dijet cross sections given by Eqs. (3.7) and (4.28), respectively. One immediately notices that the differential cross sections (3.7) and (4.28) are proportional to each other, similarly to what was seen earlier in the case of Abelian radiation in Refs. [10-12]. When calculating the SD-to-inclusive ratio, however, one notices that $\mathcal{K}_{\mathrm{SD}}$ and $\mathcal{K}_{\text {incl }}$ are functions of $\alpha$ which has to be integrated out in the corresponding cross sections. For example, using the above results with $q \rightarrow q+G$ subprocess, we obtain

$$
\begin{aligned}
\Delta \sigma_{\mathrm{SD}}^{q \rightarrow q G} \equiv & \frac{d \sigma_{\mathrm{SD}}^{q \rightarrow q G}}{d x_{G}}=\delta \int_{x_{G}}^{1} \frac{d \alpha}{\alpha^{2}} \mathcal{K}_{\mathrm{SD}}^{q \rightarrow q G}(s, \hat{s}, \alpha) \\
& \times \sum_{q, \bar{q}}\left[q\left(x_{q}, Q^{2}\right)+\bar{q}\left(x_{q}, Q^{2}\right)\right] \\
& \times \frac{4}{3} \frac{\alpha_{s}\left(Q^{2}\right)}{\pi} \int_{\rho_{\min }}^{\rho_{\max }} d \rho \rho^{3}\left\{m_{q}^{2} \alpha^{4} K_{0}^{2}(\tau \rho)\right. \\
& \left.+\left[1+(1-\alpha)^{2}\right] \tau^{2} K_{1}^{2}(\tau \rho)\right\},
\end{aligned}
$$

where

$$
x_{q}=\frac{x_{G}}{\alpha}, \quad \hat{s}=s x_{q},
$$

and $\tau=\tau(\alpha)$ is defined in Eq. (2.4), $\mathcal{K}_{\mathrm{SD}}^{q \rightarrow q G}$ is defined in Eq. (4.30), and the integration limits are $\rho_{\min } \sim 1 / Q$ and $\rho_{\text {min }} \sim 1 / E_{T, \text { min }}^{1,2}, E_{T, \text { min }}^{1,2}=5 \mathrm{GeV}$.

Analogically, for the inclusive dijet cross section for the gluon Bremsstrahlung $q \rightarrow q G$ subprocess, we write

$$
\begin{aligned}
\Delta \sigma_{\text {incl }}^{q \rightarrow q G} \equiv & \frac{d \sigma_{\text {incl }}^{q \rightarrow q G}}{d x_{G}}=\int_{x_{G}}^{1} \frac{d \alpha}{\alpha^{2}} \mathcal{K}_{\text {incl }}^{q \rightarrow q G}\left(x_{2}, \alpha\right) \sum_{q, \bar{q}}\left[q\left(x_{q}, Q^{2}\right)\right. \\
& \left.+\bar{q}\left(x_{q}, Q^{2}\right)\right] \\
& \times \frac{4}{3} \frac{\alpha_{s}\left(Q^{2}\right)}{\pi} \int_{\rho_{\min }}^{\rho_{\max }} d \rho \rho^{3}\left\{m_{q}^{2} \alpha^{4} K_{0}^{2}(\tau \rho)\right. \\
& \left.+\left[1+(1-\alpha)^{2}\right] \tau^{2} K_{1}^{2}(\tau \rho)\right\}
\end{aligned}
$$

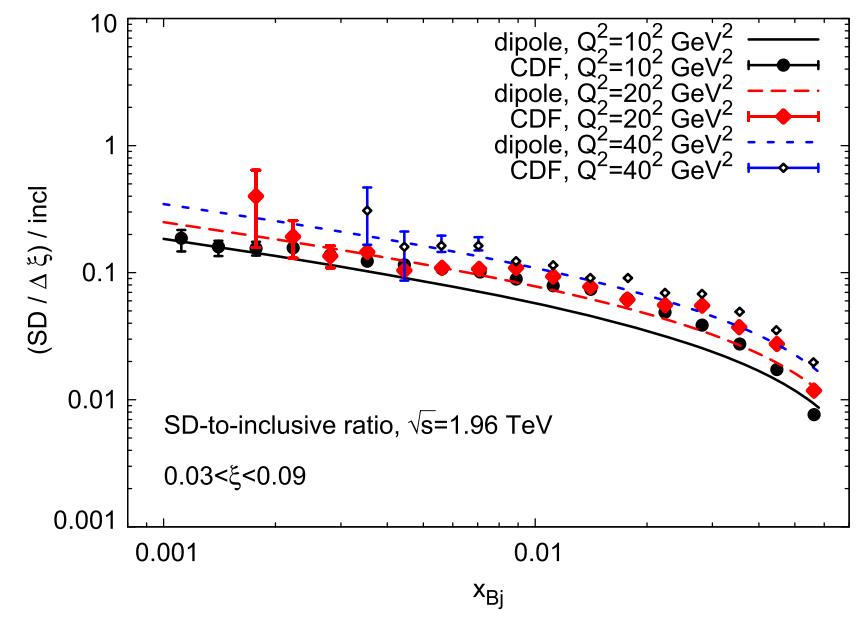

FIG. 4. The SD-to-inclusive ratio $\mathcal{R}_{\mathrm{SD} / \text { incl }}\left(x_{\mathrm{Bj}}, Q^{2}\right)$ given by Eq. (5.11) as function of $x_{\mathrm{Bj}}$ for three different values of the hard scale $Q^{2}=10^{2}, 20^{2}$ and $40^{2} \mathrm{GeV}^{2}$ in comparison to the CDF Run II data [47].

where $\mathcal{K}_{\text {incl }}^{q \rightarrow q G}$ is defined in Eq. (3.6), and $x_{2}=Q^{2} / s x_{G}$ (see also Ref. [10]). The cross sections for the gluon-initiated subprocesses, such as $G \rightarrow q \bar{q}$ and $G \rightarrow G_{1} G_{2}$, can be obtained in complete analogy to the above expressions, except that the (anti)quark densities are replaced by the gluon one.

Finally, the SD-to-inclusive ratio is written as follows

$$
\begin{aligned}
& \mathcal{R}_{\mathrm{SD} / \text { incl }} \\
& =\frac{1}{\Delta \xi} \frac{d \sigma_{\mathrm{SD}}^{q \rightarrow q G} / d x_{G}+d \sigma_{\mathrm{SD}}^{G \rightarrow q \bar{q}} / d x_{G}+d \sigma_{\mathrm{SD}}^{G \rightarrow G_{1} G_{2}} / d x_{G}}{d \sigma_{\text {incl }}^{q \rightarrow q G} / d x_{G}+d \sigma_{\text {incl }}^{G \rightarrow q \bar{q}} / d x_{G}+d \sigma_{\text {incl }}^{G \rightarrow G_{1} G_{2}} / d x_{G}}
\end{aligned}
$$

accounting for the proper phase space constraints. In Fig. 4 we show the $\mathrm{SD}$-to-inclusive ratio $\mathcal{R}_{\mathrm{SD} / \text { incl }}$ computed by using Eq. (5.11) as function of $x_{\mathrm{Bj}}$ variable for three different values of the hard scale $Q^{2}=10^{2}, 20^{2}$ and $40^{2} \mathrm{GeV}^{2}$ and compared to the corresponding CDF Run II data [47]. In addition, in Fig. 5 we show partial contributions to the $\mathrm{SD}$-to-inclusive ratio $\mathcal{R}_{\mathrm{SD} / \text { incl }}\left(x_{\mathrm{Bj}}, Q^{2}\right)$ at fixed $Q^{2}=40^{2} \mathrm{GeV}^{2}$ corresponding to $G \rightarrow G G$ (solid line), $q \rightarrow q G$ (dashed line) and $G \rightarrow q \bar{q}$ (dash-dotted line) subprocesses. Apparently, $G \rightarrow G G$ process is dominant in the SD production of dijets in the considered kinematical region.

Notice that while the GBW parametrization of the dipole cross section, Eq. (3.1), is sufficiently accurate for many applications, the DGLAP evolution within the used scale range might be not negligible. Therefore, we introduced here a scale dependence of the parameter $R_{0}$ in (3.1) in accordance with the model [38]. We found the effect rather small, but it somewhat improves agreement with data. 


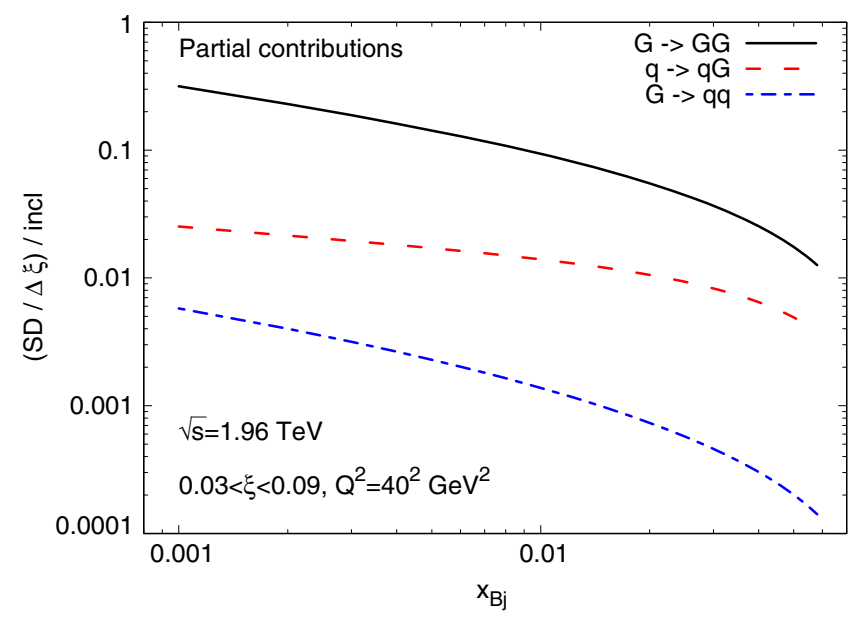

FIG. 5. The SD-to-inclusive ratio $\mathcal{R}_{\mathrm{SD} / \text { incl }}\left(x_{\mathrm{Bj}}, Q^{2}\right)$ given by Eq. (5.11) where $\sigma_{\mathrm{SD}}$ corresponds to one of the three partial contributions $G \rightarrow G G$ (solid line), $q \rightarrow q G$ (dashed line) and $G \rightarrow$ $q \bar{q}$ (dash-dotted line) as functions of $x_{\mathrm{Bj}}$ for a fixed value of the hard scale $Q^{2}=40^{2} \mathrm{GeV}^{2}$ and at Tevatron energy $\sqrt{s}=1.96 \mathrm{TeV}$.

The energy and hard scale dependences of the SD-toinclusive ratio $\mathcal{R}_{\mathrm{SD} / \text { incl }}$ are typically considered to be an important qualitative measure of the diffractive factorization breaking. Similarly to the SD Drell-Yan $[9,10]$ and gauge boson [11] production cases, an important feature of the ratio $\mathcal{R}_{\mathrm{SD} / \text { incl }}$ in the $\mathrm{SD}$ dijet production case also inconsistent with a factorization-based analysis, is its unusual energy and scale dependence shown in Fig. 6. It appears to be remarkably universal for both SD Abelian and non-Abelian types of radiation. As was discussed earlier in Refs. [14,15], the ratio $\mathcal{R}_{\mathrm{SD} / \text { incl }}$, in particular, its normalization and slopes in $\sqrt{s}$ and $Q^{2}$, is sensitive only to a particular (processdependent) linear combination of the universal dipole cross section evaluated at different separations causing an interplay between hard and soft fluctuations (see also Ref. [9]).

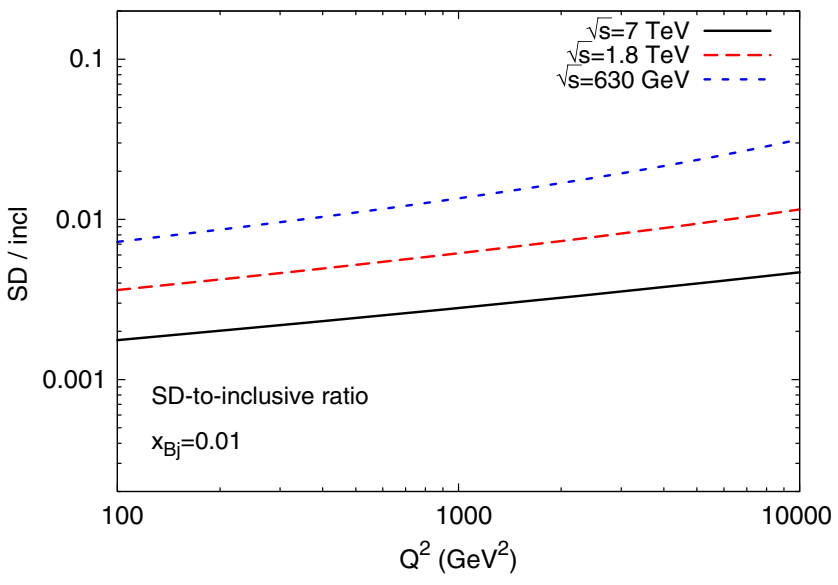

FIG. 6. The SD-to-inclusive ratio $\mathcal{R}_{\mathrm{SD} / \text { incl }}\left(x_{\mathrm{Bj}}, Q^{2}\right)$ as function of $Q^{2}$ for three different values of the c.m. energy $\sqrt{s}=630 \mathrm{GeV}, 1.8 \mathrm{TeV}$ and $7 \mathrm{TeV}$. No additional phase-space correction factor $\delta$ and no division by $\Delta \xi$ have been applied here.
Notably, the sign of these slopes is the same for all the SD reactions, that have been studied in the dipole picture so far, but it is clearly opposite to that in the existing factorizationbased predictions (cf. Ref. [49]). In this sense, the SD-toinclusive ratio can be used as an important probe for the QCD mechanism of diffraction that is essentially determined by an interplay between hard and soft interactions. As a possible direction for future studies, in order to quantify the factorization breaking effects, it would be instructive to make a more detailed comparison between the predictions of the dipole and factorization-based models.

\section{SUMMARY AND CONCLUSIONS}

In this work, we computed the inclusive and singlediffractive cross sections for dijet production in hadronhadron collisions in the dipole picture accounting for the quark $(q \rightarrow q G)$ and gluon $(G \rightarrow q \bar{q}, G G)$ excitations. Applied for the kinematics of the CDF experiment at the Tevatron, we estimated the SD-to-inclusive cross section ratio $\mathcal{R}_{\mathrm{SD} / \text { incl }}$ as function of $x_{\mathrm{Bj}}$ and hard scale of the process $Q^{2}$. Diffractive factorization is found to be severely broken for many reasons.

First, the diffractive structure functions, measured in the diagonal diffractive DIS, should not be applied to an offdiagonal hadronic diffraction, like dijet production. Such a mismatch causes dramatic effects, usually related to the rapidity gap survival probability. Working at the amplitude level in the dipole representation the gap survival factor is by default embedded into our calculation of the diffractive amplitude.

The gap survival amplitude is related to possible interactions with the spectator partons. However, we found that factorization is broken even in the case of diffractive excitation of a projectile quark, $q \rightarrow q G$, the process free of spectators. Our calculations within the dipole formalism results in a cross section falling with relative jet transverse momentum as $1 / \kappa^{6}$, while the factorization would lead to $1 / \kappa^{4}$ dependence.

Remarkably, interactions with the spectator partons in the projectile hadron, not only suppress the cross section, but also considerably increase it, giving rise to a new mechanism of diffractive dijet production. Interaction with the spectator quarks, separated by large transverse distance from the active one, causes an interplay of the long-range interactions with the spectator partons, and the hard-scale interactions with a given Fock state. A similar conclusion, which has resulted in a dynamically calculated rapidity gap survival factor derived from the modelling of multiparton interactions, has been made in Ref. [50].

The results for $\mathcal{R}_{\mathrm{SD} / \text { incl }}\left(x_{\mathrm{Bj}}, Q^{2}\right)$ exhibit an overall consistency with the data available from Tevatron. Notice that these results for non-Abelian (gluon Bremsstrahlung and splitting) types of radiation, and SD Abelian diffractive radiation (Drell-Yan $[9,10]$ ) demonstrate an interesting 
similarity in shapes and magnitudes, pointing at a universal character of the diffractive factorization breaking effects in hadronic diffraction.

\section{ACKNOWLEDGMENTS}

B. K. and I. P. are partially supported by Fondecyt Grant No. 1170319 (Chile), by Proyecto Basal FB 0821 (Chile), and by Conicyt Grant No. PIA ACT1406 (Chile). R. P. is partially supported by the Swedish Research Council, Contracts No. 621-2013-4287 and No. 2016-05996, by
CONICYT Grants No. PIA ACT1406 and No. MEC80170112, as well as by the European Research Council (ERC) under the European Union's Horizon 2020 research and innovation programme (Grant Agreement No. 668679). This work was supported in part by the Ministry of Education, Youth and Sports of the Czech Republic, Project No. LT17018. The work has been performed in the framework of COST Action CA15213 "Theory of hot matter and relativistic heavy-ion collisions" (THOR).
[1] B. Kopeliovich and B. Povh, Interplay of soft and hard interactions in nuclear shadowing at high $q^{* *} 2$ and low $x$, Z. Phys. A 356, 467 (1997).

[2] A. B. Kaidalov, Diffractive production mechanisms, Phys. Rep. 50, 157 (1979).

[3] B.Z. Kopeliovich, I. K. Potashnikova, and I. Schmidt, Diffraction in QCD, Braz. J. Phys. 37, 473 (2007).

[4] G. Ingelman and P. E. Schlein, Jet structure in high mass diffractive scattering, Phys. Lett. 152B, 256 (1985).

[5] F. Abe et al. (CDF Collaboration), Measurement of Diffractive Dijet Production at the Tevatron, Phys. Rev. Lett. 79, 2636 (1997).

[6] G. Alves, E. Levin, and A. Santoro, Diffractive production of $b \bar{b}$ in $p \bar{p}$ collision at the Tevatron, Phys. Rev. D 55, 2683 (1997).

[7] J. C. Collins, L. Frankfurt, and M. Strikman, Diffractive hard scattering with a coherent pomeron, Phys. Lett. B 307, 161 (1993).

[8] F. Yuan and K. T. Chao, Diffractive charm jet production at hadron colliders in the two gluon exchange model, Phys. Rev. D 60, 094012 (1999).

[9] B. Z. Kopeliovich, I. K. Potashnikova, I. Schmidt, and A. V. Tarasov, Unusual features of Drell-Yan diffraction, Phys. Rev. D 74, 114024 (2006).

[10] R. S. Pasechnik and B. Z. Kopeliovich, Drell-Yan diffraction: Breakdown of QCD factorisation, Eur. Phys. J. C 71, 1827 (2011).

[11] R. Pasechnik, B. Kopeliovich, and I. Potashnikova, Diffractive gauge bosons production beyond QCD factorisation, Phys. Rev. D 86, 114039 (2012).

[12] R. Pasechnik, B. Z. Kopeliovich, and I. K. Potashnikova, Diffractive Higgsstrahlung, Phys. Rev. D 92, 094014 (2015).

[13] B. Z. Kopeliovich, I. K. Potashnikova, I. Schmidt, and A. V. Tarasov, Diffractive excitation of heavy flavors: Leading twist mechanisms, Phys. Rev. D 76, 034019 (2007).

[14] R. Pasechnik, B. Kopeliovich, and I. Potashnikova, Diffractive bremsstrahlung in hadronic collisions, Adv. High Energy Phys. 2015, 1 (2015).

[15] B. Kopeliovich, R. Pasechnik, and I. Potashnikova, Hard hadronic diffraction is not hard, Int. J. Mod. Phys. E 25, 1642001 (2016).
[16] R. J. Glauber, Cross-sections in deuterium at high-energies, Phys. Rev. 100, 242 (1955).

[17] E. Feinberg and I. Ya. Pomeranchuk, High energy inelastic diffraction phenomena, Nuovo Cimento Suppl. 3, 652 (1956).

[18] M. L. Good and W. D. Walker, Diffraction disssociation of beam particles, Phys. Rev. 120, 1857 (1960).

[19] B. Z. Kopeliovich and L. I. Lapidus, Quark-parton model for hadron-nucleus interactions, Pisma Zh. Eksp. Teor. Fiz. 28, 664 (1978).

[20] B. Z. Kopeliovich, L. I. Lapidus, and A. B. Zamolodchikov, Dynamics of color in hadron diffraction on nuclei, Pisma Zh. Eksp. Teor. Fiz. 33, 612 (1981) [JETP Lett. 33, 595 (1981)].

[21] B. Z. Kopeliovich, A. Schäfer, and A. V. Tarasov, Nonperturbative effects in gluon radiation and photoproduction of quark pairs, Phys. Rev. D 62, 054022 (2000).

[22] M. G. Ryskin, A. D. Martin, and V. A. Khoze, Soft processes at the LHC. II. Soft-hard factorization breaking and gap survival, Eur. Phys. J. C 60, 265 (2009).

[23] M. G. Ryskin, A. D. Martin, and V. A. Khoze, High-energy strong interactions: From 'hard' to 'soft', Eur. Phys. J. C 71, 1617 (2011).

[24] B. Z. Kopeliovich, A. V. Tarasov, and A. Schäfer, Bremsstrahlung of a quark propagating through a nucleus, Phys. Rev. C 59, 1609 (1999).

[25] Y. V. Kovchegov and A. H. Mueller, Gluon production in current nucleus and nucleon-nucleus collisions in a quasiclassical approximation, Nucl. Phys. B529, 451 (1998).

[26] V. P. Goncalves, M. Krelina, J. Nemchik, and R. Pasechnik, Drell-Yan process in $p A$ collisions: Path-integral treatment of coherence effects, Phys. Rev. D 94, 114009 (2016).

[27] B. Kopeliovich, Soft component of hard reactions and nuclear shadowing, in Proceedings of the International Workshop XXIII on Gross Properties of Nuclei and Nuclear Excitations, Hirschegg, Austria, 1995, edited by $\mathrm{H}$. Feldmeyer and W. Nörenberg (Gesellschaft Schwerionenforschung, Darmstadt, 1995), p. 385.

[28] S. J. Brodsky, A. Hebecker, and E. Quack, The Drell-Yan process and factorization in impact parameter space, Phys. Rev. D 55, 2584 (1997). 
[29] B. Z. Kopeliovich, J. Raufeisen, and A. V. Tarasov, The color dipole picture of the Drell-Yan process, Phys. Lett. B 503, 91 (2001).

[30] E. Basso, V. P. Goncalves, J. Nemchik, R. Pasechnik, and M. Sumbera, Drell-Yan phenomenology in the color dipole picture revisited, Phys. Rev. D 93, 034023 (2016).

[31] B. Z. Kopeliovich and A. V. Tarasov, Gluon shadowing and heavy flavor production off nuclei, Nucl. Phys. A710, 180 (2002).

[32] N. N. Nikolaev, G. Piller, and B. G. Zakharov, Quantum coherence in heavy flavor production on nuclei, Zh. Eksp. Teor. Fiz. 108, 1554 (1995) [J. Exp. Theor. Phys. 81, 851 (1995)].

[33] N. N. Nikolaev, G. Piller, and B. G. Zakharov, Inclusive heavy flavor production from nuclei, Z. Phys. A 354, 99 (1996).

[34] K. J. Golec-Biernat and M. Wusthoff, Saturation effects in deep inelastic scattering at low $Q^{* *} 2$ and its implications on diffraction, Phys. Rev. D 59, 014017 (1998).

[35] B. Blaettel, G. Baym, L. L. Frankfurt, and M. Strikman, How Transparent are Hadrons to Pions?, Phys. Rev. Lett. 70, 896 (1993).

[36] L. Frankfurt, G. A. Miller, and M. Strikman, Coherent nuclear diffractive production of mini - jets: Illuminating color transparency, Phys. Lett. B 304, 1 (1993).

[37] L. Frankfurt, A. Radyushkin, and M. Strikman, Interaction of small size wave packet with hadron target, Phys. Rev. D 55, 98 (1997).

[38] J. Bartels, K. J. Golec-Biernat, and H. Kowalski, A modification of the saturation model: DGLAP evolution, Phys. Rev. D 66, 014001 (2002).

[39] R. M. Barnett et al., Particle physics summary, Rev. Mod. Phys. 68, 611 (1996).
[40] S. R. Amendolia et al. (NA7 Collaboration), A measurement of the space-like pion electromagnetic form-factor, Nucl. Phys. B277, 168 (1986).

[41] B. Z. Kopeliovich, I. K. Potashnikova, B. Povh, and I. Schmidt, Evidences for two scales in hadrons, Phys. Rev. D 76, 094020 (2007).

[42] M. Glück, E. Reya, and A. Vogt, Radiatively generated parton distributions for high-energy collisions, Z. Phys. C 48, 471 (1990).

[43] M. Glück, E. Reya, and A. Vogt, Dynamical parton distributions of the proton and small $x$ physics, Z. Phys. C 67, 433 (1995).

[44] M. Glück, E. Reya, and A. Vogt, Dynamical parton distributions revisited, Eur. Phys. J. C 5, 461 (1998).

[45] B.Z. Kopeliovich, I. K. Potashnikova, and I. Schmidt, Large rapidity gap processes in proton-nucleus collisions, Phys. Rev. C 73, 034901 (2006).

[46] Yu. M. Kazarinov, B. Z. Kopeliovich, L. I. Lapidus, and I. K. Potashnikova, Triple Regge phenomenology in the reaction $p+p \rightarrow p+X$, Zh. Eksp. Teor. Fiz. 70, 1152 (1976) [Sov. Phys. JETP 43, 598 (1976)].

[47] T. Aaltonen et al. (CDF Collaboration), Diffractive Dijet production in $\bar{p} p$ collisions at $\sqrt{s}=1.96 \mathrm{TeV}$, Phys. Rev. D 86, 032009 (2012).

[48] B. Z. Kopeliovich, H. J. Pirner, I. K. Potashnikova, K. Reygers, and I. Schmidt, Pion-pion cross section from proton-proton collisions at the LHC, Phys. Rev. D 91, 054030 (2015).

[49] M. Luszczak, R. Maciula, A. Szczurek, and I. Babiarz, Singlediffractive production of dijets within the $k_{t}$-factorization approach, Phys. Rev. D 96, 054018 (2017).

[50] C. O. Rasmussen and T. Sjöstrand, Hard diffraction with dynamic gap survival, J. High Energy Phys. 02 (2016) 142. 